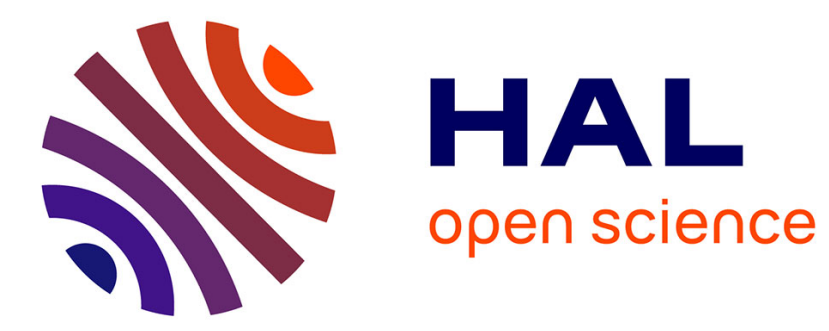

\title{
Slip activities in polycrystals determined by coupling DIC measurements with crystal plasticity calculations
} Adrien Guery, François Hild, Félix Latourte, Stéphane Roux

\section{To cite this version:}

Adrien Guery, François Hild, Félix Latourte, Stéphane Roux. Slip activities in polycrystals determined by coupling DIC measurements with crystal plasticity calculations. International Journal of Plasticity, 2016, 81, pp.249-266. 10.1016/j.ijplas.2016.01.008 . hal-01303523

\section{HAL Id: hal-01303523 \\ https://hal.science/hal-01303523}

Submitted on 21 Apr 2016

HAL is a multi-disciplinary open access archive for the deposit and dissemination of scientific research documents, whether they are published or not. The documents may come from teaching and research institutions in France or abroad, or from public or private research centers.
L'archive ouverte pluridisciplinaire HAL, est destinée au dépôt et à la diffusion de documents scientifiques de niveau recherche, publiés ou non, émanant des établissements d'enseignement et de recherche français ou étrangers, des laboratoires publics ou privés. 


\title{
Slip activities in polycrystals determined by coupling DIC measurements with crystal plasticity calculations
}

\author{
Adrien Guery ${ }^{\mathrm{a}, \mathrm{b}}$, François Hild ${ }^{\mathrm{a}}$, Félix Latourte ${ }^{\mathrm{b}}$, Stéphane Roux ${ }^{\mathrm{a}}$ \\ ${ }^{a}$ LMT-Cachan (ENS Cachan/CNRS/University Paris-Saclay) \\ 61 avenue du Président Wilson, F-94235 Cachan (FRANCE) \\ ${ }^{b} E D F R \mathcal{G} D$, Site des Renardières, avenue des Renardières - Ecuelles, F-77818 Moret-sur-Loing (FRANCE)
}

\begin{abstract}
The role of crystallographic grain size and microstructure of an austenitic stainless steel (A316LN) is studied via in-situ mechanical testing in an SEM and digital image correlation to measure displacement and strain fields. The latter ones are directly measured on a mesh supported by the grain boundaries as imaged by EBSD. This experimental analysis is applied to five microstructures of the same cast material but subjected to different heat treatments. Differences in the strain distributions that can be attributed to the grain size are observed. The full-field measurements allow in turn for a direct, local and quantitative comparison with crystal plasticity finite element simulations of the same experiments based on the same mesh. A weak correlation between the plastic strain and the Schmid's factors, which are computed with macroscopic or even local stress evaluations, emphasizes the role of inter-grain strain incompatibilities or grain boundaries. However, a good agreement in the major slip system evaluation between experimental observation and computations is observed.
\end{abstract}

Keywords: Crystal plasticity, Digital Image Correlation, Grain size effect, Slip activity

\section{Introduction}

In several industrial fields, multiscale mechanical models have been developed to better understand and predict the performance of materials and to improve the links between microstructural and mechanical properties. The present study focuses on a face-centered cubic 316LN austenitic stainless steel, which is widely used for high temperature structural components in power plants such as Pressurized Water Reactors (PWRs) because of its corrosion resistance, 
fracture toughness and creep performance [1].

If mean-field homogenization techniques are often sufficient to derive macroscopic properties from microscopic ones, they are unable to provide the local mechanical fields that are needed to study degradation mechanisms occurring at the microstructural scale (e.g., stress corrosion cracking [2-4] or intergranular fracture [5-8]). Such fields can, for example, be computed using full-field methods based upon fast-Fourier transforms [9-12] now available for finite strain calculations [13], or finite element methods as reviewed by Roters et al. [14].

In this context, the behavior of the grain or twin boundaries is not yet fully understood and remains a challenging research area. For instance, the interactions of dislocations with the interfaces in polycrystals depend on the interface structure itself $[15,16]$, which is affecting the stress levels. The experimentally observed lattice curvature at grain boundaries [16-18] is successfully accounted for in strain gradient plasticity models [19-22] or higher-order continua models [23-26], which are motivated by the need for describing Geometrically Necessary Dislocations (GNDs) [27]. Among possible applications of such models, the study of plasticity in the vicinity of crack tips [18], Hall-Petch effects [23, 28-33] or even grain size effects on plastic strain localization patterns [34] can be cited. The latter phenomena are observed at scales of about one micrometer, consistent with the predictions of several numerical works [30, 35-38].

In this study, it is first proposed to explore the grain size effects at a mesoscopic scale using Digital Image Correlation (DIC) from images acquired during in-situ tensile tests in a Scanning Electron Microscope (SEM). DIC provides spatially dense experimental information in polycrystals [39], more particularly for studying grain boundary effects [40, 41]. Several studies have shown the possibility of performing kinematic measurements at the microstructural scale, for instance, using an Atomic Force Microscope (AFM) [42] or SEM [43, 44]. These imaging means allow for the mapping of strain fields at the surface of polycrystals with high spatial resolutions $[43,44]$. In particular, strain localizations have been shown to be related to grain orientations $[45,46]$. These studies lead, for instance, to a correlation between these localizations and the Schmid's factors. Such kinematic measurements also provide a way to analyze the slip system activity (whose slip traces are directly observed on the surface) and more specifically the slip transmission across grain boundaries [47, 48]. Other applications of DIC at microstructural 
scales deal with features in the vicinity of the crack tip $[49,50]$, to determine global fracture mechanics parameters [51], or to assess the cumulated plastic strain close to a growing fatigue crack [52]. Recent advances in crystal plasticity modeling have shown the opportunities of confronting these experimental investigations with simulations in terms of textures [9], of relating fields to the microstructure [40, 41, 53, 54], of validating models [55-57], or even parameter identification [39].

In the present case and contrary to all the previous studies, the displacement field is based on a mesh taking as support the grain boundaries of the observed surface. This is made possible by using Electron Back-Scattered Diffraction (EBSD) data, which give access to the crystallographic orientations, and requires a change of coordinate system. While in similar studies such transformation is achieved using micro-indentations $[39,52]$, a registration method using DIC is chosen herein.

Traditionally, experimental slip system activities are compared with those predicted by the Schmid's factors based on a far field stress projection [58]. Using this approach, a lack of correlation in the polycrystalline case is attributed to grain neighboring and localization effects [59]. In addition, subsurface grains have a known influence of the kinematic surface response of polycrystals $[60,61]$, and this challenge has been recently mostly tackled by studying oligocrystals [5658], bicrystals [41], or directionally solidified polycrystals [62]. However, such experimental conditions are not possible in the present case of an industrial material for which the grain size dimension is small as compared to the sample thickness.

Displacement fields are measured on meshes built at the free surfaces of five different tensile polycrystalline specimens of grade $316 \mathrm{LN}$ stainless steel. The slip activities are then explored by resorting to full-field finite element simulations of the experiments, considering a local stress projector outperforming far field projectors as employed in Ref. [58]. Using these techniques, correlations between model and experiments are finally discussed. 


\section{Material and experimental facilities}

\subsection{Material}

The material of the present study is a $316 \mathrm{LN}$ austenitic stainless steel of face centered cubic (FCC) crystal lattice. The tensile specimens are machined from a 60-mm thick rolled plate also utilized within the AFGRAP project $[63,64]$, which was subjected to a solution treatment at $1050-1150{ }^{\circ} \mathrm{C}$ followed by water quenching. Preliminary optical microscopy and EBSD analyses have revealed the presence of less than 0.1 vol. $\%$ of $\delta$-ferrite in the plate core and no texture was observed. To avoid the ferritic phase, the sampling is conducted in the plate skins where the material is fully austenitic. After solution treatment, a typical microstructure with heterogeneous grain sizes is observed, namely, the average size is of the order of $70 \mu \mathrm{m}$ while a small fraction of small grains down to $5 \mu \mathrm{m}$ in size coexists with some larger grains up to $200 \mu \mathrm{m}$. The grain shapes are equiaxed and large sized grains are sometimes divided into twins. The chemical composition of the material is given in Table 1.

Table 1: Chemical composition of the studied 316LN stainless steel plate in weight $\%$

\begin{tabular}{|c|c|c|c|c|c|c|c|}
\hline $\mathrm{Fe}$ & $\mathrm{Cr}$ & $\mathrm{Ni}$ & $\mathrm{Mo}$ & $\mathrm{Mn}$ & $\mathrm{Si}$ & $\mathrm{N}$ & $\mathrm{Co}$ \\
\hline bal. & 17.5 & 12.5 & 2.47 & 1.7 & 0.37 & 0.072 & 0.04 \\
\hline \hline $\mathrm{C}$ & $\mathrm{P}$ & $\mathrm{Cu}$ & $\mathrm{Ti}$ & $\mathrm{Nb}$ & $\mathrm{Ta}$ & $\mathrm{B}$ & $\mathrm{S}$ \\
\hline 0.027 & 0.024 & 0.01 & 0.005 & 0.005 & 0.003 & 0.0005 & 0.0002 \\
\hline
\end{tabular}

In this study, five different specimens, denoted $A_{70}, B_{10}, C_{10}, D_{50}$ and $E_{1000+}$, machined from this 316LN plate are considered. They have different average grain sizes (indicated by their designation subscript) determined by EBSD in a chosen Region Of Interest (ROI) detailed in the next section, due to different treatments:

- sample $A_{70}$ is machined from the plate in its as-received state. The mean grain size is of the order of $70 \mu \mathrm{m}$,

- samples $B_{10}$ and $C_{10}$ are taken from the plate after $60 \%$ cold-rolling followed by 1-hour heat treatment at $900{ }^{\circ} \mathrm{C}$. The resulting mean grain size is about $10 \mu \mathrm{m}$, 
- sample $D_{50}$ is machined from the plate after $60 \%$ cold-rolling followed by 1-hour heattreatment at $1100{ }^{\circ} \mathrm{C}$. The resulting mean grain size is about $50 \mu \mathrm{m}$,

- sample $E_{1000+}$ is taken from the plate after 264 -hour heat treatment at $1200{ }^{\circ} \mathrm{C}$. The resulting microstructure shows millimetric grains.

\subsection{Microstructure characterization and in-situ tensile tests}

In this study, a Zeiss Supra 55 SEM is used with a TSL OIM analysis software for EBSD measurements. An FEI Quanta FEG 600 SEM is used for imaging purposes. A miniaturized tensile testing device is introduced in the SEM chamber so that in-situ tensile tests can be imaged all along the loading history. EBSD measurements are performed with an acceleration voltage of $25 \mathrm{kV}$ and a working distance of $14 \mathrm{~mm}$. For imaging, both Secondary Electron (SE) EverhartThornley detector and Backscattered Electron (BSE) detector in Z-contrast are used, with an acceleration voltage of $10 \mathrm{kV}$ and a working distance of $14 \mathrm{~mm}$. An image definition of $2048 \times$ 1887 pixels and a dynamic range of 16 bits are chosen.

The imaged gray level speckle marking evolved upon straining due to surface variations, namely, the deformation of the crystalline lattice and/or the accumulation of slip bands on the observed surface, which is related to the electromagnetic imaging environment. As these contrast variations appear more significantly with the SE mode than the BSE mode, the latter is chosen for imaging purposes during any in-situ tensile test to make DIC calculations easier. The SE mode is used to actually observe these surface variations at the end of tensile tests (see Section 5.3).

For each tensile test, imaging conditions are the same but with three different magnifications. Table 2 summarizes the measurement conditions. For samples $A_{70}$ and $B_{10}$ the same ROI size is selected, even though the grain size is different. Conversely, samples $B_{10}$ and $C_{10}$ have the same grain size but different ROI sizes are chosen. Last, samples $D_{50}$ and $E_{1000+}$ are analyzed with the same ROI size. It is worth noting that the ROI covers only few twins in one single grain for sample $E_{1000+}$.

Figure 1 shows the grain size distributions in the ROI of each specimen (except $E_{1000+}$ ). These distributions are obtained from the grain partition based on the EBSD measurements. One may note that the distributions of samples $B_{10}$ and $C_{10}$ are similar. Conversely, the distributions of 
Table 2: DIC measurements conditions for the five different specimens

\begin{tabular}{|c|c|c|c|c|c|}
\hline Specimen & $\begin{array}{c}\text { Pixel size } \\
(\mathrm{nm})\end{array}$ & $\begin{array}{c}\text { ROI size } \\
\left(\mu \mathrm{m}^{2}\right)\end{array}$ & $\begin{array}{c}\text { Mean grain size } \\
(\mu \mathrm{m})\end{array}$ & $\begin{array}{c}\text { Mean grain size } \\
(\text { pixel })\end{array}$ & $\begin{array}{c}\text { Number of grains } \\
\text { in the ROI }\end{array}$ \\
\hline$A_{70}$ & 150 & $200 \times 200$ & 70 & 470 & 14 \\
\hline$B_{10}$ & 160 & $200 \times 200$ & 10 & 60 & 551 \\
\hline$C_{10}$ & 80 & $100 \times 100$ & 10 & 120 & 117 \\
\hline$D_{50}$ & 280 & $400 \times 400$ & 50 & 180 & 1 \\
\hline$E_{1000+}$ & 270 & $400 \times 400$ & $>1000$ & $>3700$ & 59 \\
\hline
\end{tabular}

samples $A_{70}$ and $D_{50}$ are more irregular, which may be due to the lower number of grains in their ROIs.

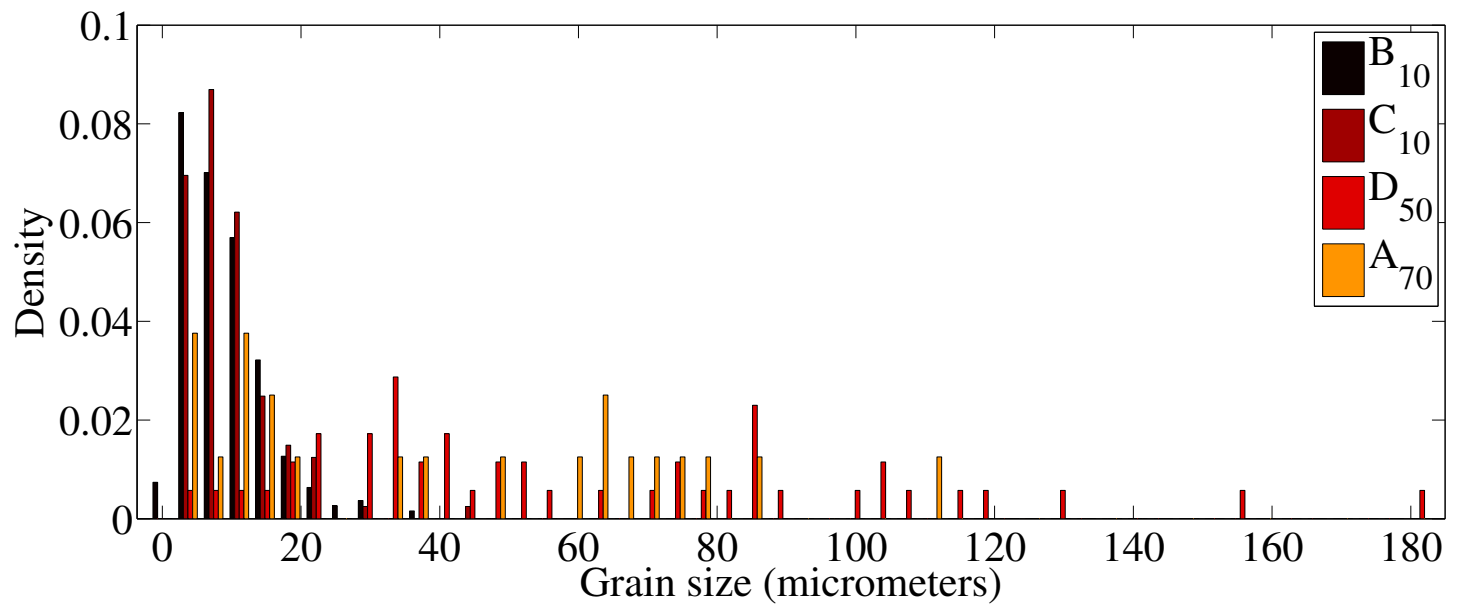

Figure 1: Grain size distributions in the ROI of the studied specimens

\section{Microstructure-based mesh for DIC measurements}

\subsection{Kinematic measurements based on SEM images}

Kinematic measurements by digital image correlation require a gray level speckled pattern with local contrasts as high as possible. In the present case, the raw image of the material does not provide such contrast. It is chosen to print an artificial speckle provided by a computer-generated 
random pattern using a microphotolithographic technique [65]. The lithography process is conducted in a specific area on the surface of an in-situ specimen. The resulting pattern is made of gold speckles whose thickness is of the order of $20 \mathrm{~nm}$. Because the speckle is designed for DIC analyses, the physical size of the spots lies in the range of 6 to 20 pixels (i.e., between 0.5 and $2.8 \mu \mathrm{m}$ ) for a center to center distance in the range of 30 to 50 pixels (i.e., between 2.7 and $10 \mu \mathrm{m})$. To allow for EBSD measurements over the region of interest, the surface is initially polished with cloths and diamond solutions down to $1 \mu \mathrm{m}$, and finished with a 50-nm colloidal silica suspension.

From a sequence of SEM images acquired during an in-situ tensile test, displacement fields are measured with a continuous Galerkin-based DIC procedure [66, 67]. The image registration is based on the conservation of the gray levels between two consecutive images, namely a first one in the reference configuration, $f$, and a second one in the deformed configuration, $g$, expressed as

$$
f(\boldsymbol{x})=g(\boldsymbol{x}+\boldsymbol{u}(\boldsymbol{x}))
$$

where $\boldsymbol{x}$ is the vector coordinate of pixels in the reference configuration, and $\boldsymbol{u}$ the sought displacement field. The minimization of the quadratic norm of the difference of gray levels $\rho_{c}^{2}=\|f(\boldsymbol{x})-g(\boldsymbol{x}+\boldsymbol{u}(\boldsymbol{x}))\|_{\mathrm{ROI}}^{2}$ is performed successively where, at iteration $n$, the displacement field is corrected by incremental displacement amplitudes $\left\{\delta \boldsymbol{u}^{(n)}\right\}$, which are the solution to linear systems

$$
[\boldsymbol{M}]\left\{\boldsymbol{\delta} \boldsymbol{u}^{(n)}\right\}=\left\{\boldsymbol{b}^{(n)}\right\}
$$

where $\{\boldsymbol{\delta} \boldsymbol{u}\}$ is the vector gathering the corrections of the displacement amplitudes $u_{k}$ associated with the basis of displacement fields $\boldsymbol{\psi}_{k}$ providing the discretized displacement field $\boldsymbol{u}(\boldsymbol{x})$

$$
\boldsymbol{u}(\boldsymbol{x})=\sum_{k} u_{k} \boldsymbol{\psi}_{k}(\boldsymbol{x})
$$

The DIC matrix $[\boldsymbol{M}]$ and vector $\left\{\boldsymbol{b}^{(n)}\right\}$ are expressed as 


$$
M_{k l}=\sum_{R O I}\left(\psi_{k} \cdot \nabla f\right)(x)\left(\psi_{l} \cdot \nabla f\right)(x)
$$

and

$$
b_{k}=\sum_{R O I}\left(f-\tilde{g}^{(n)}\right)(\boldsymbol{x})\left(\boldsymbol{\psi}_{k} \cdot \nabla f\right)(\boldsymbol{x})
$$

where $\tilde{g}^{(n)}$ is the deformed image corrected by the current estimate of the displacement field.

Several choices are possible for the kinematic basis $\boldsymbol{\psi}_{k}$. For instance, finite element shape functions $[66,68]$ may be considered or enriched bases to account for localized phenomena [69, 70]. In this study, it is chosen to take advantage of the crystallographic information that is available on the surface of the analyzed specimen. The aim is to build a finite element mesh taking as support the microstructure (i.e., grain and twin boundaries) and to overlay it on the first image of a sequence of SEM acquisitions during in situ tensile tests in order to perform DIC measurements thereafter.

The noise of SEM imaging estimated by correlating two pictures with no motion applied between two acquisitions is relatively high (i.e., greater than $2 \%$ of the dynamic range). In addition, the discretization of the displacement field has to be fine enough to capture plastic strain localizations. For those reasons, mechanical regularization appears to be beneficial to assist DIC calculations. For that purpose, in the spirit of Tikhonov regularization of ill-posed problems [71], it is proposed to include an additional cost function to the DIC gray levels functional [72]. It consists of introducing the equilibrium gap $\Phi_{m}^{2}$ penalizing deviations from a solution to a linear elastic homogeneous problem $[\boldsymbol{K}]\{\boldsymbol{u}\}=\{\mathbf{0}\}$ expressed as

$$
\Phi_{m}^{2}=\{\boldsymbol{u}\}^{t}[\boldsymbol{K}]^{t}[\boldsymbol{K}]\{\boldsymbol{u}\}
$$

where $[\boldsymbol{K}]$ is the stiffness matrix associated with inner nodes. An additional term is to be introduced to specifically deal with boundary nodes [67]. These gaps introduce two regularization lengths (i.e., one for the inner nodes and another one for the boundary nodes) weighting the equilibrium gaps against the image registration, which are both chosen to be equal to four times the characteristic length of the mesh, which corresponds to the mean length of the element edges, in 
this study. This regularization is a low-pass mechanical filter, where the regularization lengths are acting as cut-off wavelengths. It is not intended to be representative of the actual material behavior. Table 3 gives the standard measurement resolutions, in terms of displacement and strain, obtained with the presented DIC procedure for each specimen by registering two pictures with no applied load between the two acquisitions. The root mean square value of the measured displacement and strain fields due to SEM noise leads to the corresponding standard measurement uncertainties. It can be noted that their levels are quite similar for the different specimens.

Table 3: DIC standard measurement uncertainties for the five studied microstructures with the chosen correlation parameters

\begin{tabular}{|c|c|c|}
\hline Specimen & Displacement uncertainty & Strain uncertainty \\
\hline$A_{70}$ & $4.3 \mathrm{~nm} / 2.9 \times 10^{-2}$ pixel & $1.1 \times 10^{-3}$ \\
\hline$B_{10}$ & $5.9 \mathrm{~nm} / 3.7 \times 10^{-2}$ pixel & $1.5 \times 10^{-3}$ \\
\hline$C_{10}$ & $2.2 \mathrm{~nm} / 2.8 \times 10^{-2}$ pixel & $1.2 \times 10^{-3}$ \\
\hline$D_{50}$ & $7.1 \mathrm{~nm} / 2.5 \times 10^{-2}$ pixel & $1.1 \times 10^{-3}$ \\
\hline$E_{1000+}$ & $4.4 \mathrm{~nm} / 1.8 \times 10^{-2}$ pixel & $9.6 \times 10^{-4}$ \\
\hline
\end{tabular}

\subsection{Change of coordinate system and unstructured mesh}

This part is devoted to the presentation of the method to build an unstructured mesh consistent with microstructural interfaces focusing on the case of specimen $E_{1000+}$ for illustration purposes. EBSD measurements provide a map of the microstructure boundaries for the region of interest of the analyzed specimen. A misorientation greater than $15^{\circ}$, respectively about $60^{\circ}$, is chosen to define a grain boundary or a twin boundary, respectively. Figure 2(a) shows the boundaries of the microstructure, which consists of a single grain with a few embedded twins of sharp polygonal shape. This map is used as a support for building an unstructured mesh. Therefore, it is essential that the mesh, which is first expressed in the EBSD frame, be accurately transported in the SEM images frame. For that purpose, the Image Quality (IQ) map is used. For EBSD measurements using the OIM analysis software, the IQ factor estimates the quality of the diffraction pattern, 
which is based on the sum of the detected peaks in the Hough transform [73]. A gray scale mapping of IQs over the ROI would be white when the IQ is high and black otherwise. In this study, EBSD measurements are performed over gold speckles. Because the quality of the diffraction pattern is low at each point the electron beam traverses gold, the speckles appear in black in the IQ map (Figure 2(b)).

The transformation $\boldsymbol{X}(\boldsymbol{x})$ that maps the EBSD coordinate system $\boldsymbol{x}$ onto that of SEM images $\boldsymbol{X}$ is measured via DIC between the IQ map and an SE image acquired before mechanical loading (Figure 2(c)). The EBSD map is obtained by scanning a flat surface that is tilted by an angle of 70 degrees. Any slight misalignment of the EBSD acquisition system, which may be induced by the control system of the SEM stage, leads to a spatial distortion that makes the transformation $\boldsymbol{X}(\boldsymbol{x})$ non trivial (see Ref. [74] for more details on this issue). To assess this transformation and to correct it, image processing is required. First, the gray scale of the IQ map is reversed so that the pattern appears bright on a dark background. Then, a variation of gray levels with the microstructure, more visible in the IQ map than in the SE image, is to be attenuated. Image registration is performed with the suited filter giving more weight to high spatial frequencies of the gray level variations (i.e., corresponding to the speckles). The transformation can now be measured by DIC using the previously presented formulation. Since a large transformation occurs between the two images (i.e., a displacement range of 70 pixels), the algorithm is initialized with a coarse displacement solution obtained using a quadratic interpolation for each component, itself estimated from manually selecting a few remarkable points.

The correlation residuals $\rho_{c}$ are shown in Figure 3. The root mean square (RMS) value is of the order of $10 \%$ of the reference image dynamic range. Slight fluctuations appear at the position of dots, which are due to the initial difference of gray levels in the two images since the registration between the two images is successfully achieved (otherwise the speckles would appear twice).

Any EBSD map or specifically the microstructure boundaries and the crystal orientation maps in the present study can now be shown in the SEM image coordinate system. Once overlaid the twin boundaries are segmented into polygons. The path between two consecutive detected triple points is chosen to be discretized into a finite number of wires. A mesh is generated with 3-noded 


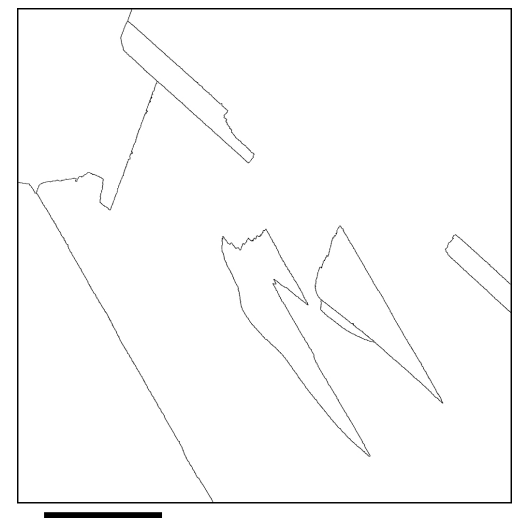

(a)

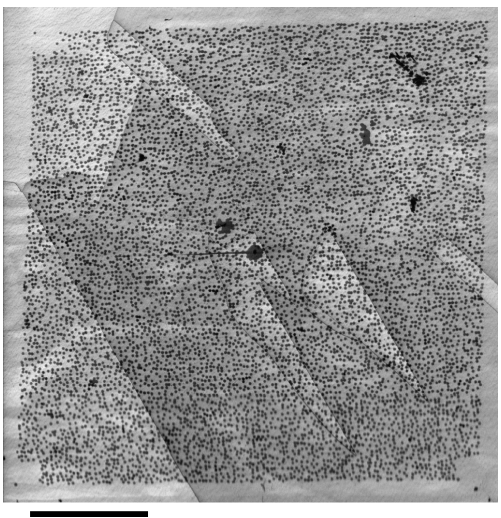

(b)

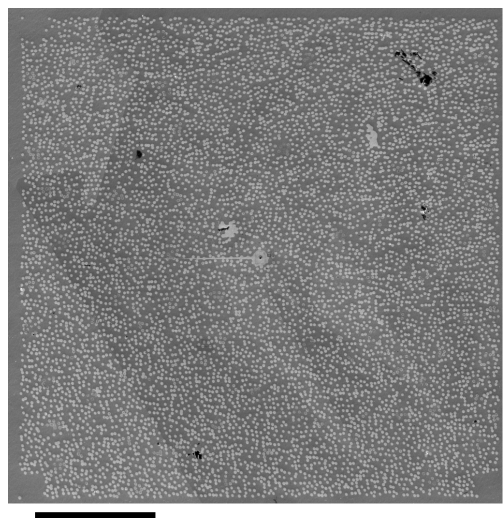

(c)

Figure 2: Twin boundaries (a) and gray scale IQ map (b) of the region of interest obtained by EBSD of specimen $E_{1000+}$. SEM imaging of the same region of interest with SE detector (c). The scale bar is $100 \mu \mathrm{m}$ long

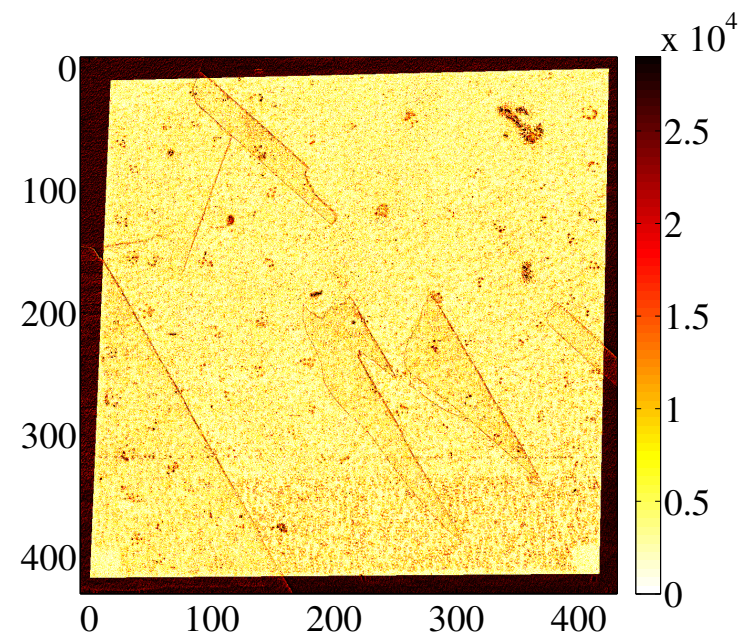

Figure 3: Absolute value of the correlation residuals expressed in gray levels corresponding to the DIC measurement between the IQ map (Figure 2(b)) and the SE image (Figure 2(c)). The dynamic range of the registered images is 16 bits. The axes are expressed in micrometers

triangular elements taking as support these polygons. The resulting mesh made of about 20-pixel (or 5- $\mu \mathrm{m}$ ) long elements is shown in Figure 4. Since the mesh is created as conforming to the microstructure boundaries the local crystal orientation can be selected for each element. 

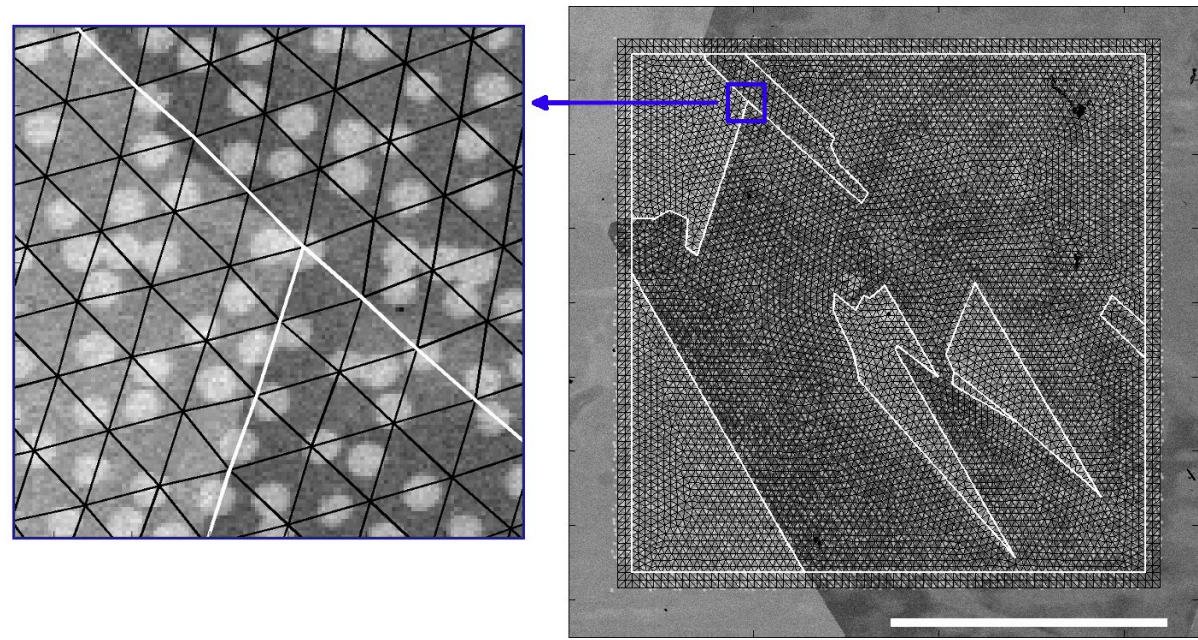

Figure 4: Unstructured mesh compatible with the underlying microstructure overlaid on the BSE image of the region of interest. The scale bar is $200 \mu \mathrm{m}$ long

\section{Results of DIC measurements in polycrystals and grain size effects}

The series of images acquired during the tensile tests are now processed with the previously presented DIC algorithm (see Equation (2)). Even though the BSE mode is used for imaging purposes, gray level changes occur with topographic variations. As a consequence, DIC measurements are performed in two steps. First, an updated scheme is applied. At the end of each calculation, an incremental displacement field is computed, the deformed image becomes the reference image for the next calculation and the total displacement is the sum of all increments. The mesh is updated too by moving the node positions with the measured nodal displacement at the end of each calculation. Second, the measured displacement fields are used to initialize a non-updated scheme (i.e., the reference image and the mesh are kept unchanged for the calculations with all deformed images). This second step allows the measurement uncertainties not to be cumulated since at each step the total displacement field is directly computed.

DIC measurements are performed for the five specimens. In each case a gold pattern is deposited and an unstructured mesh taking as support the microstructure boundaries is built. For all used magnifications, the characteristic length of the mesh is about 20 pixels (i.e., between 1.6 and $5.6 \mu \mathrm{m}$ ) and the cut-off length associated with the elastic regularization is 80 pixels (i.e., between 
6.4 and $22 \mu \mathrm{m})$. It is worth noting that the present regularization length is short compared to the mean grain size, and hence conclusions based on features of the displacement field pertaining to larger scale than the regularization length, i.e., not influenced by the mechanical regularization, result from the sample imaging only and are considered trustworthy.

Strains are evaluated for each finite element of the mesh by differentiation of the shape functions and computing the nominal components. Figure 5 shows the longitudinal component of the Green-Lagrange strain field for each microstructure, for a mean tensile strain up to $5 \%$. For each experiment, strain bands occur early on during the tensile test while only their magnitude increase thereafter. These strain localizations occur in the vicinity of grain boundaries as well as within the grains.

The statistical distribution of strain magnitude does not reveal a very significant difference that could be attributed to the grain size. In contrast, the image resolution (i.e., physical size of one pixel) has a visible impact. Thus it seems that the localized nature of the strain fields is such that it cannot be captured in an objective way with the present approach. In addition, a large number of grains is needed to establish firm conclusions and this is in conflict with exploring a large interval of grain sizes.

Since different microstructures are tested, the effect of the grain size on the macroscopic load is first considered. In Figure 6, the macroscopic stress-strain curve of the five tensile tests are compared. The Hall-Petch effect [75] applies and is reproducible, namely, the yield stress is observed to increase linearly with the inverse of the square root of the grain size, together with an increase of hardening (Figure 7). A linear interpolation of coefficient 0.96 demonstrates the good correlation between experimental data and the Hall-Petch relationship.

These experimental results are now to be compared with numerical simulations using a crystal plasticity model. In the following, only microstructure $A_{70}$ will be considered.

\section{Using crystal plasticity to interpret the experiments}

Simulations corresponding to the experimental tensile test are performed using the finite element software Code_Aster [76]. The crystal plasticity law chosen in this study has been proposed by Méric et al. [77]. It implies plastic flow, isotropic and kinematic hardening laws 


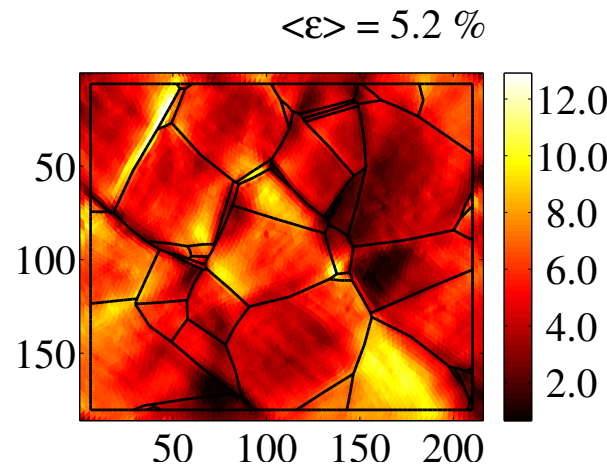

(a)

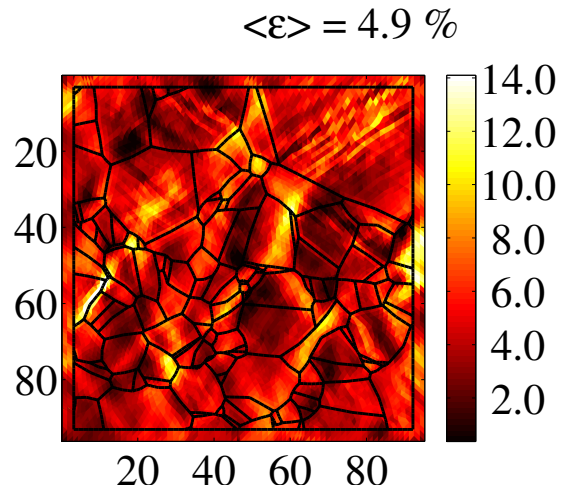

(c)

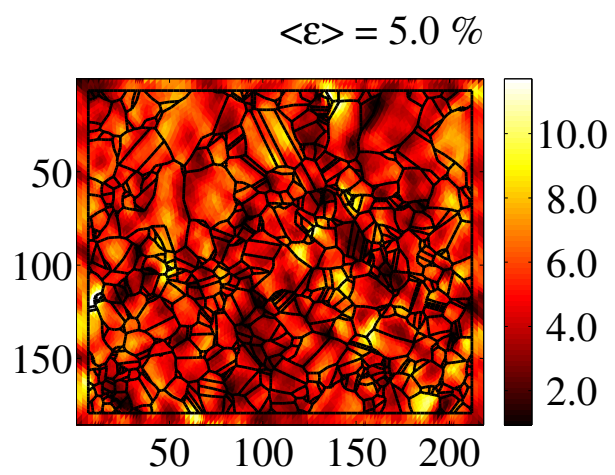

(b)

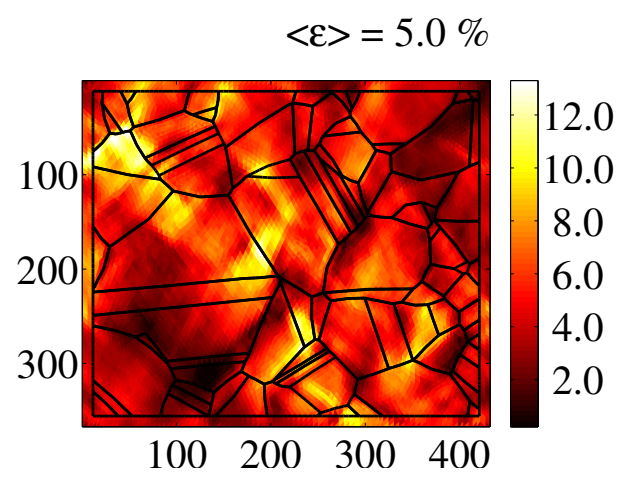

(d)

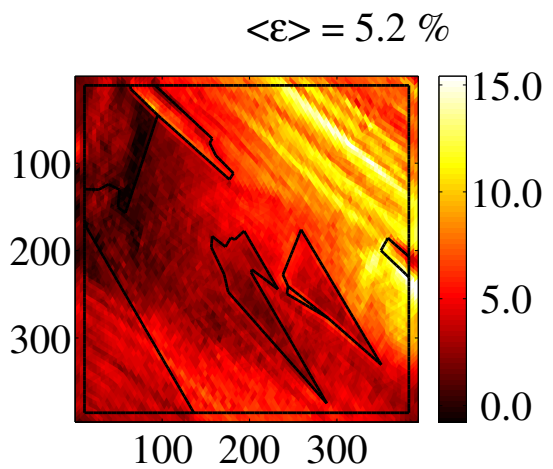

(e)

Figure 5: Strain field along the horizontal (tensile) direction calculated from DIC measurements for a mean tensile strain $\langle\epsilon\rangle$ up to $5 \%$, for the microstructures denoted $A_{70}$ (a), $B_{10}$ (b), $C_{10}$ (c), $D_{50}$ (d) and $E_{1000+}$ (e). The axes are expressed in micrometers. The microstructure boundaries are shown as black lines on these fields. An animated figure is displayed in the on-line version only 


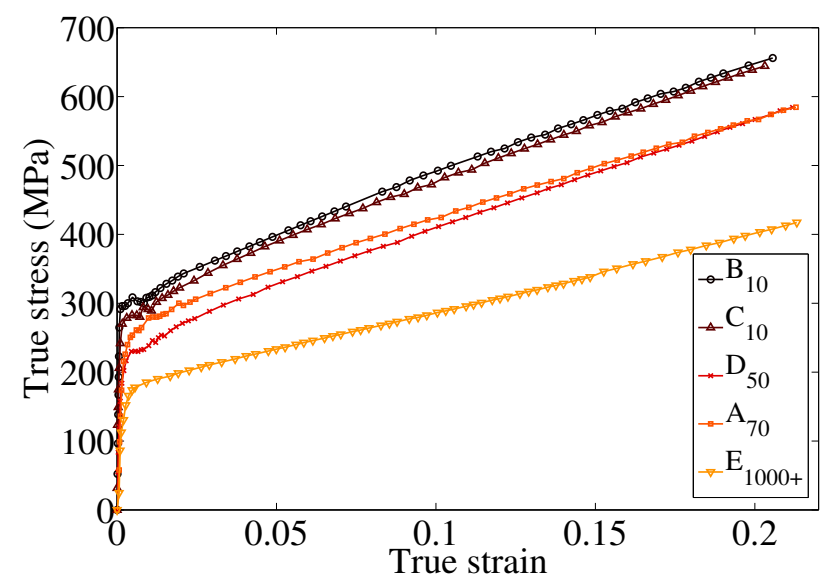

Figure 6: Experimental macroscopic stress-strain curve for the microstructures denoted $A_{70}, B_{10}, C_{10}, D_{50}$ and $E_{1000+}$

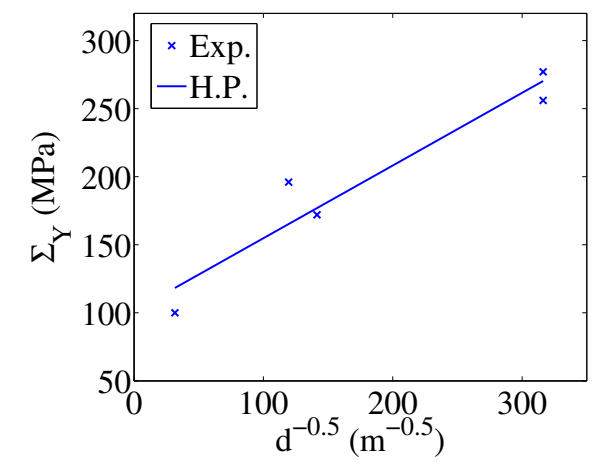

Figure 7: Identification of the Hall-Petch (H.P.) law from the experimental yield stresses obtained for the different tensile tests

expressed for each slip system (see AppendixA). Let us recall that the studied austenitic steel is an FCC material with twelve slip systems, which are listed in Table 4, indexed by $s$ and defined by a normal $\boldsymbol{n}_{s}$ to the slip plane and a slip direction $\boldsymbol{m}_{s}$. The three parameters associated with isotropic hardening are identified by homogenization [78] from the experimental macroscopic stress-strain data shown in Figure 6. The other parameters are set to values previously identified using cyclic loadings [79].

The mesh of the simulations is built from that initially used for DIC analyses. The crystal plasticity law implemented in the finite element code requires a 3D model. Consequently, 
Table 4: Adopted notation for FCC slip systems

\begin{tabular}{|c|c|c|c|c|c|c|c|c|c|c|c|c|}
\hline Normal vector $\boldsymbol{n}_{s}$ & \multicolumn{3}{|c|}{ (111) } & \multicolumn{3}{|c|}{$(1 \overline{1} 1)$} & \multicolumn{3}{|c|}{ (1̄11) } & \multicolumn{3}{|c|}{ (īî1) } \\
\hline Slip direction $\boldsymbol{m}_{s}$ & [1]01] & [0̄11] & [1110] & [1̄01] & [011] & [110] & [0̄11] & [110] & [101] & [1110] & [101] & {$[011]$} \\
\hline System index $s$ & 1 & 2 & 3 & 4 & 5 & 6 & 7 & 8 & 9 & 10 & 11 & 12 \\
\hline
\end{tabular}

because the microstructure under the surface is unknown, the initial 2D mesh is extruded perpendicular to the observed surface. This choice appeared the least arbitrary and the more realistic solution for simulating the experimental microstructure unknown in the volume compared to other methods using for instance Voronoi tessellations [60]. Since the mesh thickness in that direction appears to have a minor influence on the pattern of strain localization [39], the mesh chosen for the simulations is extruded to a distance equal to its characteristic length. The triangular prisms are then cut into 4-noded tetrahedra so that the final 3D mesh (usually referred to as "quasi-2D mesh") has one element through the thickness. Its thickness corresponds to about 20 pixels (i.e., between 1.6 and $5.6 \mu \mathrm{m}$ ), which is small compared to the specimen thickness $(1 \mathrm{~mm})$ and the mean grain size.

Conversely, experimental boundary conditions are necessary for a valid description of the substrate effects and do have a significant influence on the strain pattern $[39,80]$. Such conditions are more realistic than homogeneous boundary conditions. It is worth noting that DIC is only capable of measuring in-plane displacements while the simulation may predict an out-ofplane component. This has no consequence on the comparison performed in this work between simulations and measurements. The out-of-plane displacements appeared to have negligible consequences in the present case for macroscopic strain levels less than $5 \%$ or even for macroscopic strains close to $9 \%$ in similar experimental conditions [42].

The direct link between DIC and simulations via the common surface mesh allows the experimentally measured displacements to be prescribed with their time history as Dirichlet boundary conditions on the nodes of the edges of the ROI without any additional interpolation since both meshes are identical. The boundary conditions on the edges are not only prescribed on the sur- 
face nodes but uniformly in the thickness. In addition, zero displacements along the normal of the surface are prescribed on the back face. The simulations are performed up to a mean tensile strain of $4 \%$, and with the assumption of small strain levels. Initial experimental orientations obtained by EBSD are prescribed. In this study, averaged experimental orientations per grain are considered since no lattice curvature are observed inside the grains on the EBSD inverse pole figures for any of the studied samples. Let us stress however that the simulation allows the lattice orientation to be updated.

\subsection{Schmid's factors}

All theoretical descriptions of polycrystal plasticity require the resolved shear stress to be assessed on each slip system. These microstructural factors are of importance to the study of damage accumulation in polycrystals under fatigue conditions. For instance, persistent slip bands, which induce fatigue crack initiation, are shown to appear where the highest Schmid's factors are reached [81, 82]. Since the Schmid's factors are linked with mechanical heterogeneities under cyclic loading conditions, the relevance of correlating such microstructural factors with strain heterogeneities is investigated. For a given microstructure, the crystallographic orientations give precious information about slip system incompatibilities and thus the potential strain pattern for a considered loading condition. It is proposed to assess the slip activity from the Schmid's factors. For that purpose, the resolved shear stress on each slip system $s$ from a material standpoint is given by

$$
\tau_{s}=\sigma: \mu_{s}
$$

where the Schmid's tensor $\boldsymbol{\mu}_{s}$ of each slip system $s$ is expressed with the local crystal orientation as

$$
\boldsymbol{\mu}_{s}=\frac{1}{2}\left(\boldsymbol{m}_{s}^{*} \otimes \boldsymbol{n}_{s}^{*}+\boldsymbol{n}_{s}^{*} \otimes \boldsymbol{m}_{s}^{*}\right)
$$

with

$$
\boldsymbol{m}_{s}^{*}=\boldsymbol{R} \boldsymbol{m}_{s}, \quad \boldsymbol{n}_{s}^{*}=\boldsymbol{R} \boldsymbol{n}_{s}
$$


where $\boldsymbol{R}$ is the rotation matrix built with the local crystal orientation. The simplest approach assumes a uniform stress $\sigma_{0}$ in the loading direction $e_{L}$

$$
\boldsymbol{\sigma}=\sigma_{0} \boldsymbol{e}_{L} \otimes \boldsymbol{e}_{L}
$$

which allows the resolved shear stress to be expressed as a function of the Schmid's factors $f_{s}$

$$
\tau_{s}=\sigma_{0} f_{s}
$$

with

$$
f_{s}=\left(e_{L} \otimes e_{L}\right): \mu_{s}=\left(m_{s}^{*} \cdot e_{L}\right)\left(n_{s}^{*} \cdot e_{L}\right)
$$

However, this result relies on the strong assumption that the crystal orientation has no influence on local stresses, namely, it ignores orientation incompatibilities that lead to local heterogenous stress fields $[14,83]$. Thus, a second approach is to assess the local stress tensor to estimate the 'local' Schmid's factors $f_{s}^{l}$. The local stress tensor $\boldsymbol{\sigma}$ at each time step of the finite element simulation is considered. To keep a similar expression of the resolved shear stress (11), Von Mises' equivalent stress at each material point $\sigma_{e q}$ is used

$$
\tau_{s}=\sigma_{e q} f_{s}^{l}
$$

with

$$
f_{s}^{l}=\frac{1}{\sigma_{e q}}\left(\boldsymbol{\sigma} \cdot \boldsymbol{n}_{s}^{*}\right) \cdot \boldsymbol{m}_{s}^{*}
$$

On the edges of the ROI, because experimental displacements are applied as boundary conditions, the simulated stress field is corrupted by measurement uncertainties, and $f_{s}^{l}$ as well. For both approaches, the experimental orientations averaged over each grain have been considered. In Figure 8, the 'macroscopic' Schmid's factors $f_{s}$ are compared with the 'local' counterparts, considering slip system $s=10$ for illustration purposes. This comparison can be performed for different levels of loading, but the 'local' Schmid's factors appeared to notably change only in 
the transitional regime (i.e., for the macroscopic tensile strain ranging from 0 to $1 \%$ ). It can be noted that highlighted grains in which the slip system under consideration is mainly activated according to the 'local' factor are quite different from those mainly activated according to the 'macroscopic' factor and the difference is significant for each considered time step. The same analyses can be carried out for each slip system.

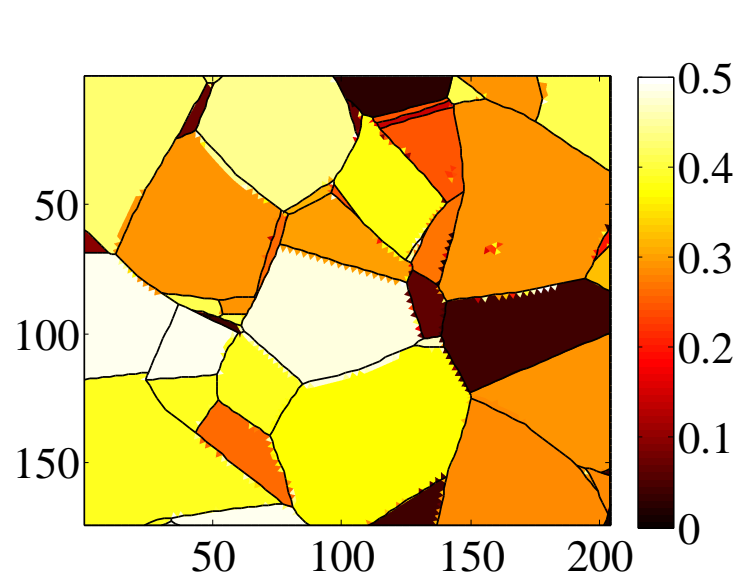

(a)

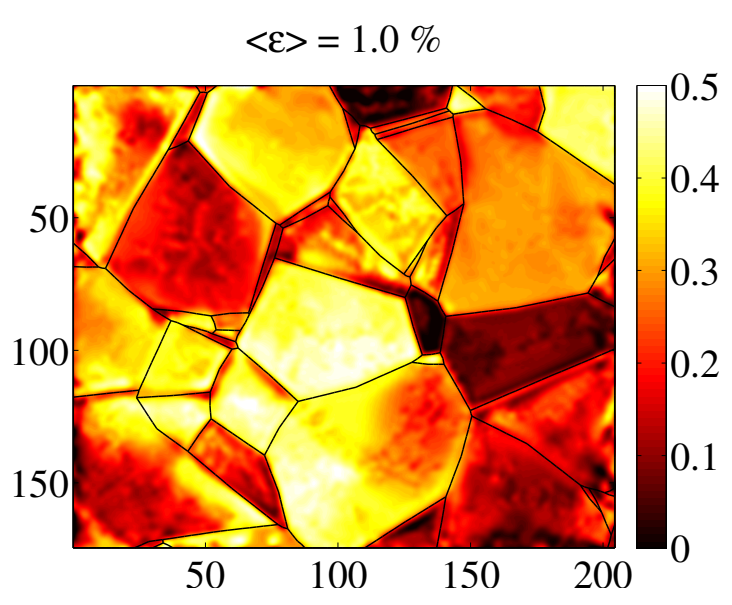

(b)

Figure 8: Schmid's factor for slip system $s=10$ computed by assuming a uniform tensile stress along the horizontal direction (a), and from the local simulated stress fields for a mean tensile strain $\langle\epsilon\rangle$ up to $1 \%$ (b). The spatial axes are expressed in micrometers. The microstructure boundaries are shown as black lines on these fields. An animated figure (b) is displayed in the on-line version only

This result is to be expected since the 'macroscopic' factors do not consider local stress heterogeneities due to orientation incompatibilities nor stress changes over time. The field of the system index $s_{I}$ of the highest 'local' Schmid's factor is shown in Figure 9(b) for a mean tensile strain of $1 \%$. It can be directly compared to that obtained using the 'macroscopic' Schmid's factors (Figure 9(a)) or even to that obtained from the main shear strain predicted by the finite element simulation (Figure 9(c)). If similarities can be observed with the latter the 'macroscopic' Schmid's factors approach offers the poorest information without intragranular variation and the field obtained from the 'local' Schmid's factors appears much more scattered. The latter can be explained by the fact that the full stress tensor is used to compute the Schmid's factors, each of its component may fluctuate locally, and because it corresponds to an instantaneous assessment 
while the shear strain approach considers the loading history. This poor agreement shows that the influence of neighboring grains on local slip activity, which is not incorporated in the approach using global Schmid's factors, should be taken into account for reaching a presumably more accurate description.

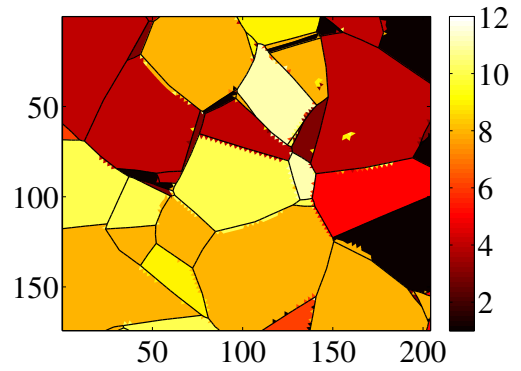

(a)

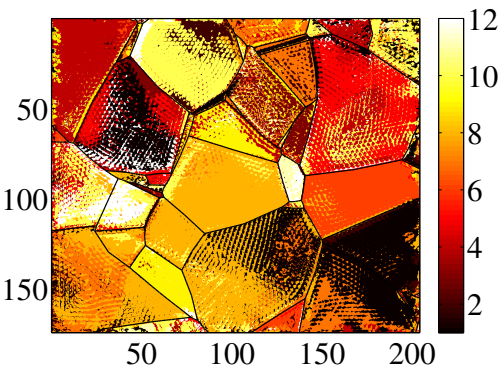

(b)

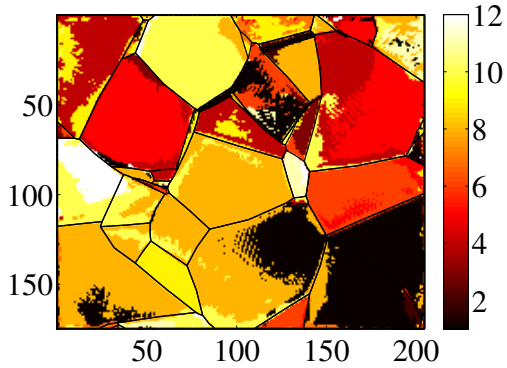

(c)

Figure 9: Field of index $s_{I}$ of the highest 'macroscopic' Schmid's factor (a). Fields of index $s_{I}$ of the highest 'local' Schmid's factor (b), and of the highest shear strain (c) for a mean tensile strain of $1 \%$. The spatial axes are expressed in micrometers. The microstructure boundaries are shown as black lines on these fields

\subsection{Plastic glide along slip systems}

It is now proposed to look at plastic glide along slip systems from the DIC measurements in a similar way as in a previous study on BCC materials [84]. The viscoplastic strain rate is assumed to be only due to plastic glide along slip systems, which is written as

$$
\dot{\boldsymbol{\epsilon}}_{p}=\sum_{s=1}^{12} \dot{\gamma}_{s} \boldsymbol{\mu}_{s}
$$

where $\dot{\gamma}_{s}$ denotes the shear strain rate on slip system $s$. From this relationship, knowing the in-plane displacement gradients from displacement measurements, an experimental assessment of plastic glide is sought. Therefore, some hypotheses need to be made. First, the experimental total strain rate $\dot{\boldsymbol{\epsilon}}$ is considered to be only composed of a viscoplastic part $\dot{\boldsymbol{\epsilon}}_{p}$ since the elastic part appeared negligible (or comparable to the DIC resolution in the experiments, see Table 3), so that

$$
\dot{\boldsymbol{\epsilon}}=\dot{\boldsymbol{\epsilon}}_{e}+\dot{\boldsymbol{\epsilon}}_{p} \approx \dot{\boldsymbol{\epsilon}}_{p}
$$


Since small perturbations are considered (in particular, small rotations), Equation (15) is integrated over time

$$
\boldsymbol{\epsilon}_{p}=\sum_{s=1}^{12} \gamma_{s} \boldsymbol{\mu}_{s}
$$

Further, DIC measurements yield only 2D (i.e., in-plane) displacement gradients, while 3D components are required in Equation (17). The assumptions of material incompressibility and that the normal axis to the image plane $z$ is an eigen direction are made, leading to only one component of the strain tensor along $z$ expressed as

$$
\epsilon_{z z} \approx-\epsilon_{x x}-\epsilon_{y y}
$$

The system expressed in Equation (17) cannot be inverted because it involves twelve unknowns $\gamma_{s}$ for only nine equations. However, only few slip systems over the twelve candidates are generally activated [47]. To illustrate this point, Figure 10(a) shows the twelve shear strains at one material point of the simulation during the entire loading history. In this example, only four slip systems have been locally activated.

Let us consider the four main activated slip systems, which are denoted as $s_{I}, s_{I I}, s_{I I I}$ and $s_{I V}$, and are identified by simulation at each material point and at each time step (i.e., for each SEM image acquisition), such that

$$
\left\{s_{I}, s_{I I}, s_{I I I}, s_{I V}\right\} \in[1,12] \quad|\quad| \gamma_{s_{I}}|\geq| \gamma_{s_{I I}}|\geq| \gamma_{s_{I I I}}|\geq| \gamma_{s_{I V}}|\geq| \gamma_{s_{j \neq I I I I I I I I, I V\}}} \mid
$$

and the function $\eta\left(\gamma_{s}\right)$, which is representative of each slip system activity relative to the total plastic activity, is defined as

$$
\eta\left(\gamma_{s}\right)=\frac{\left|\gamma_{s}\right|}{\sum_{i=1}^{12}\left|\gamma_{i}\right|}
$$

The distribution of $\eta\left(\gamma_{s}\right)$ calculated for the four main shear strains over all the Gauss points for a mean tensile strain of $4 \%$ is shown in Figure 10(b) as cumulative distribution functions. These results show that the four main slip systems account, on average, for $95 \%$ of the plastic activity. 
Figure 10(c) shows the change over time of the mean value and corresponding standard deviation of these distributions. It can be noted that the slip system activity fluctuates at the beginning of the tensile test (i.e., for a macroscopic tensile strain less than $2 \%$ ) before stabilizing. The fourth main shear strain appears not to exceed on average $10 \%$ of the total slip activity.

As a consequence, it is assumed that the slip activity is restricted to only the four main activated systems, which allows the system expressed in Equation (17) to be inverted. The change over time of the field of the first main system index $s_{I}$ identified by simulations is shown in Figure 11(a). It is interesting to note that two or three different systems are generally active at the same time for each grain. At each material point, the corresponding four main shear strains are now determined from the experimentally estimated strain tensor. The first 'experimental' main shear strain field $\gamma_{s_{I}}^{e}$ is compared to the simulated one $\gamma_{s_{I}}^{c}$ in Figure 11. Both simulation and experiment lead to very similar patterns of strain localization over time. The small difference lies in a higher scatter of $\gamma_{s_{I}}$ in the experimental case, which can be explained by the fact that only the first four activated systems are combined to build the experimental strain tensor or by the Schmid's tensors $\boldsymbol{\mu}_{s}$ being unchanged over time for the experimental approach. This almost perfect agreement tends to validate the simulation of the experiments with the chosen crystal plasticity law. In the following, results showing a more debatable agreement between experimental observation and computations are presented. 


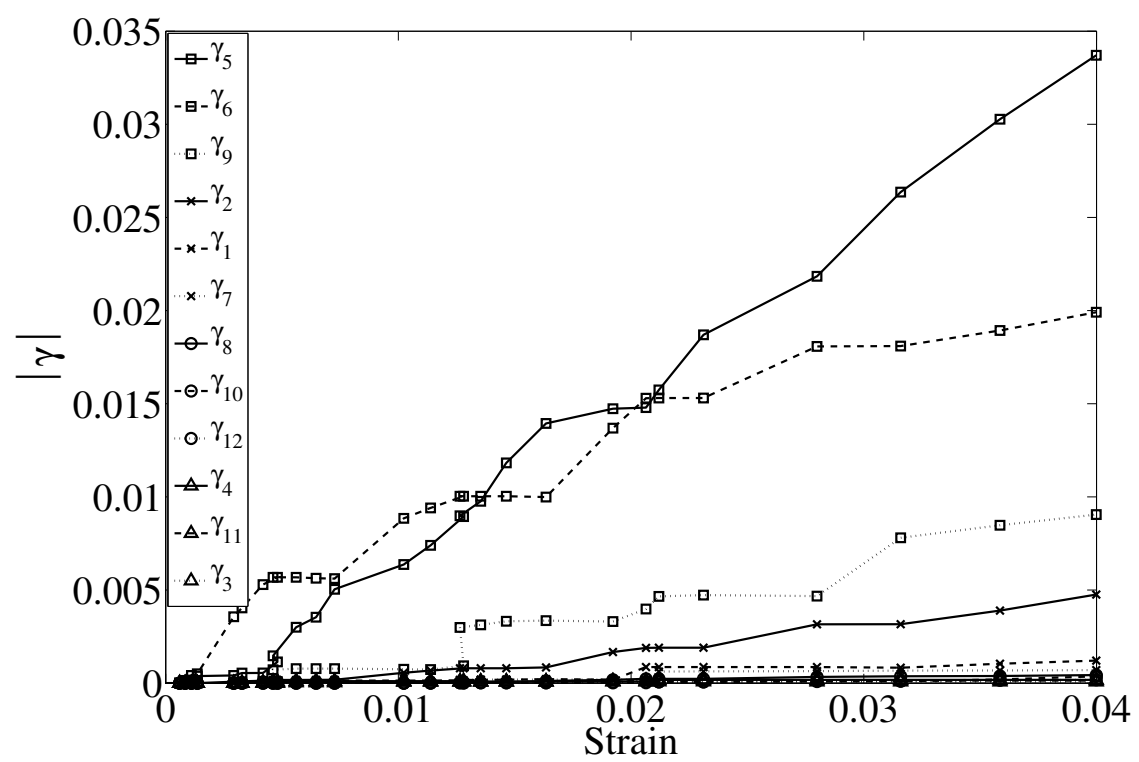

(a)

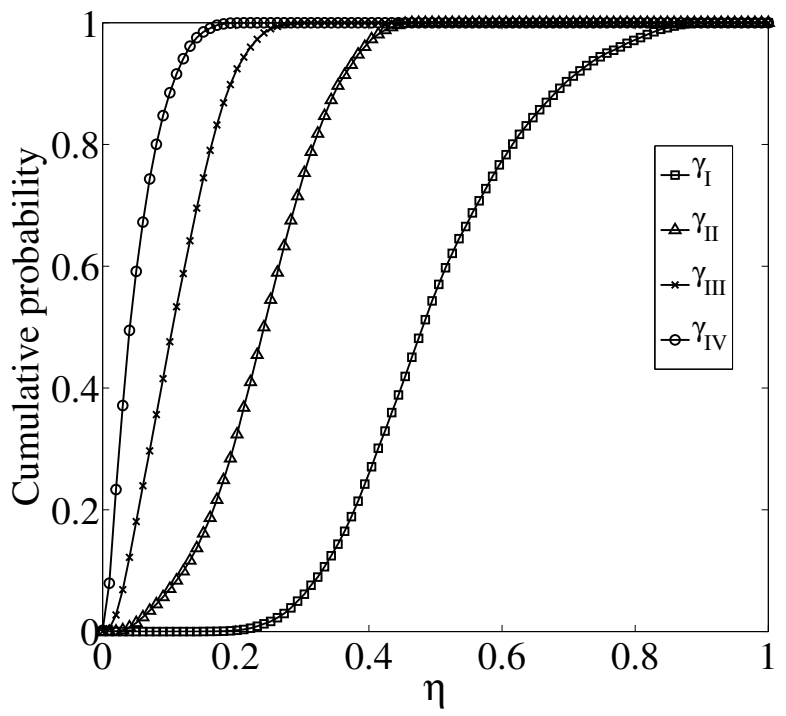

(b)

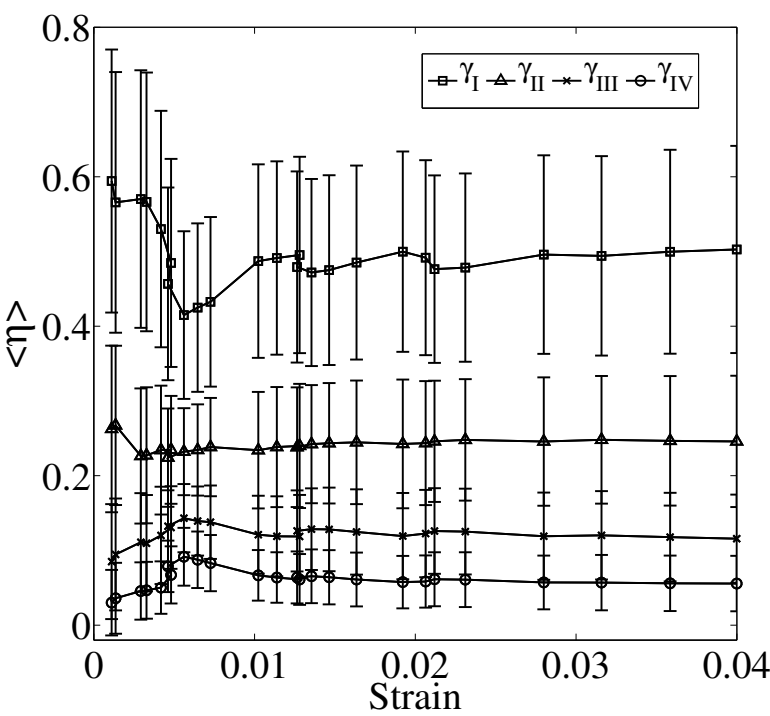

(c)

Figure 10: Change of the simulated absolute value of the twelve shear strains $\gamma_{s}$ at one material point (a). Cumulative distribution functions for the field $\eta$ of the four main shear strains for a mean tensile strain of $4 \%$ (b). Change of the mean simulated value of these distributions (c). The vertical bars indicate the standard deviations of the distributions 


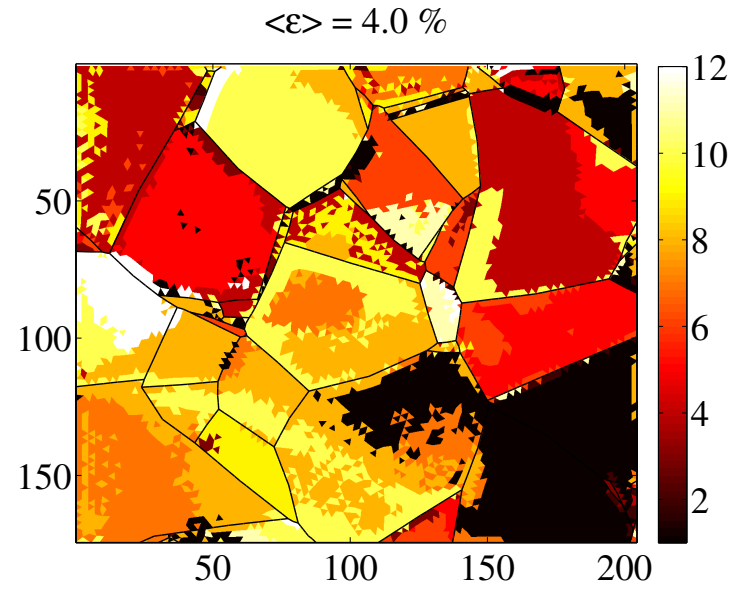

(a)

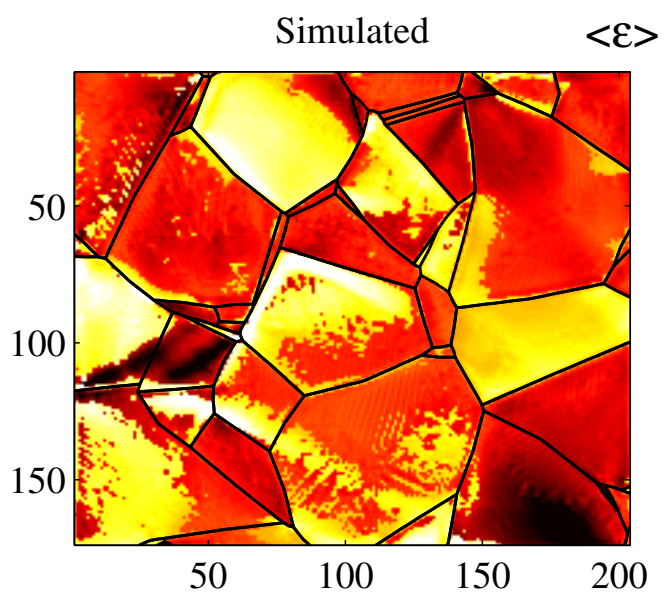

$$
=4.0 \% \quad \text { Experimental }
$$
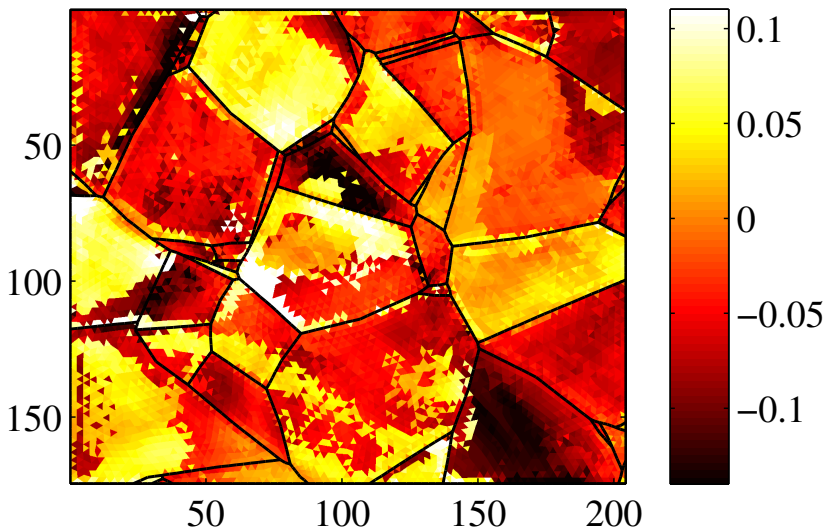

(b)

Figure 11: Field of indices of the main system $s_{I}$ for a mean tensile strain $\langle\epsilon\rangle$ in the range of $0.1 \%$ to about $4 \%$ (a). Simulated shear strain field $\gamma_{s_{l}}^{c}$ and experimental shear strain field $\gamma_{s_{I}}^{e}$ for a mean tensile strain $\langle\epsilon\rangle$ up to $4 \%$ (b). The spatial axes are expressed in micrometers. The microstructure boundaries are shown as black lines on these fields. An animated figure is displayed in the on-line version only 


\subsection{Slip traces on the surface}

During the in-situ tensile tests, the initially polished surfaces are progressively marked by slip bands resulting from activated slip systems that intercept the external surface. These bands are easily observed in SE mode, which is the reason why the BSE mode is chosen for DIC analyses. It is proposed to simulate the intensity and orientation of these bands. From the simulation of the experimental tests, only the two most activated slip systems contributing to out-of-plane extrusions are selected

$$
\left\{s_{I}, s_{I I}\right\} \in[1,12] \quad|\quad| p_{s_{I}}^{n}|\geq| p_{s_{I I}}^{n}|\geq| p_{s_{j \neq I I I I I I I I I}^{n}} \mid
$$

where $p_{s}$ is the cumulative shear strain magnitude of slip system $s$, and $p_{s}^{n}$ its projection considering the sample surface normal

$$
p_{s}=\int\left|\dot{\gamma}_{s}\right| d t
$$

with

$$
p_{s}^{n}=p_{s} \boldsymbol{e}_{z} \cdot \boldsymbol{m}_{s}
$$

The intersection of the first slip system $s_{I}$ of normal $\boldsymbol{n}_{s_{I}}$ with the external surface, which is assumed to be planar over time of normal $\boldsymbol{e}_{z}$, is the vector $\boldsymbol{t}_{s_{I}}$ expressed as

$$
\boldsymbol{t}_{s_{I}}=\boldsymbol{n}_{s_{I}} \times \boldsymbol{e}_{z}
$$

where the operator $\times$ is the vector product. Vector $\boldsymbol{t}_{s_{I I}}$ is obtained in a similar manner. In Figure 12(a), these two vectors $\boldsymbol{t}_{s_{I}}$ and $\boldsymbol{t}_{s_{I I}}$ are drawn as segments of length proportional to the value of $p_{s_{I}}^{n}$, respectively $p_{s_{I I}}^{n}$, for a mean tensile strain of $4 \%$. One SE image of the ROI has only been acquired at the end of test, for a mean tensile strain of $22 \%$. By extrapolating the results shown in Figure 10(c), the slip activity is assumed not to change notably when the mean tensile strain varies from $4 \%$ to $22 \%$. The image $g(\boldsymbol{x})$ has been corrected by the displacement measured by DIC, leading to $g(\boldsymbol{x}+\boldsymbol{u}(\boldsymbol{x}))$, which is shown in Figure 12(b) for a Lagrangian comparison with 
the previous numerical approach (i.e., the orientation of the slip traces are represented in the undeformed coordinate system). Some enlarged areas are detailed in Figures 12(c) to 12(j), which focus on experimentally observed single or double slip, close to or far from grain boundaries.

No clear correlation can be made between numerical and experimental observations (e.g., location or the nature of slip). For example, single slip observed in Figure 12(d) is correctly predicted by the simulations (Figure 12(c)), whereas for the similar situation of Figure 12(j), the double slip suggested by the simulation is not observed experimentally. Thus, even if the primary slip system is well predicted (Figure 11) the simulation differs in some places to the observed slip traces. Similar results were obtained by monitoring the slip traces using atomic force microscopy [85] with a relatively similar modeling approach. Yet the comparisons were not presented for individual slip systems but only for the different slip families of a hexagonal titanium and for a small number of grains. The differences between the simulation and the experiment may not only be due to the lack of knowledge of the underlying microstructure in the bulk but also to a constitutive model error. The fact that the SE image corresponds to a much more advanced state of loading than the simulation can explain the differences too even if the slip system activity seems not to fluctuate beyond a macroscopic tensile strain of $2 \%$ (Figure 10(c)).

\section{Conclusion and perspectives}

In this paper, the effect of grain size on the plastic behavior of $316 \mathrm{LN}$ austenitic stainless steel is studied via in-situ mechanical test within an SEM. The displacement fields are measured via digital image correlation based on a finite-element mesh that is itself tailored to the specimen microstructure as observed via EBSD. The mean grain size has a marked effect on the macroscopic yield stress and hardening. In addition, the strain fields are markedly heterogeneous with numerous shear bands either aligned with or interrupted by grain boundaries.

To relate the plastic strain to the grain crystallographic orientation, the Schmid's factors are locally computed based on two different prescriptions for the local stress. Although a local evaluation of the stress provides a better correlation between the Schmid's factors and plastic strains, the agreement remains rather poor. Thus when polycrystalline structural effects are not accounted 


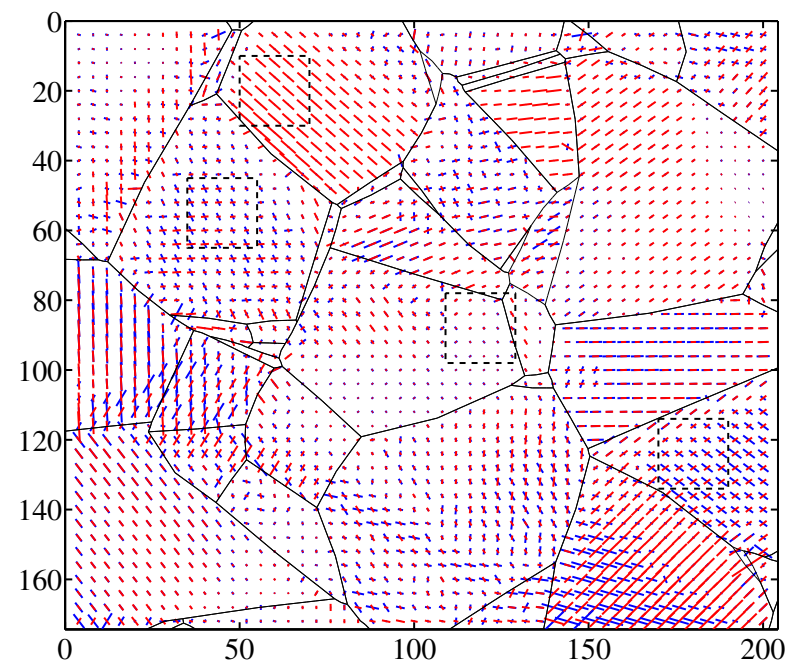

(a)

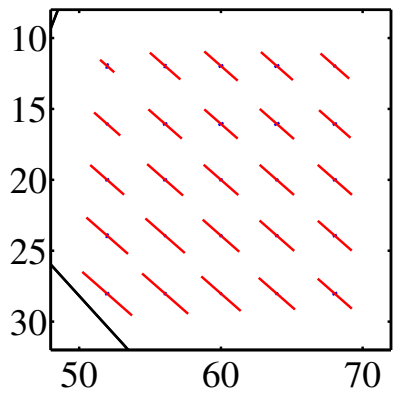

(c)

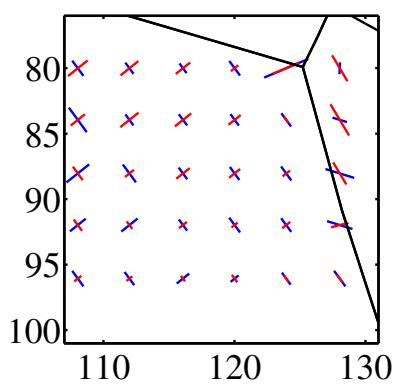

(g)

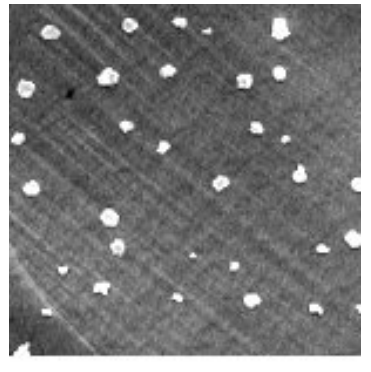

(d)

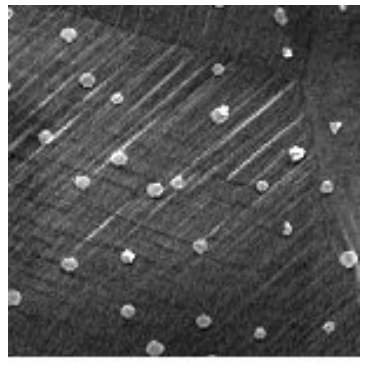

(h)

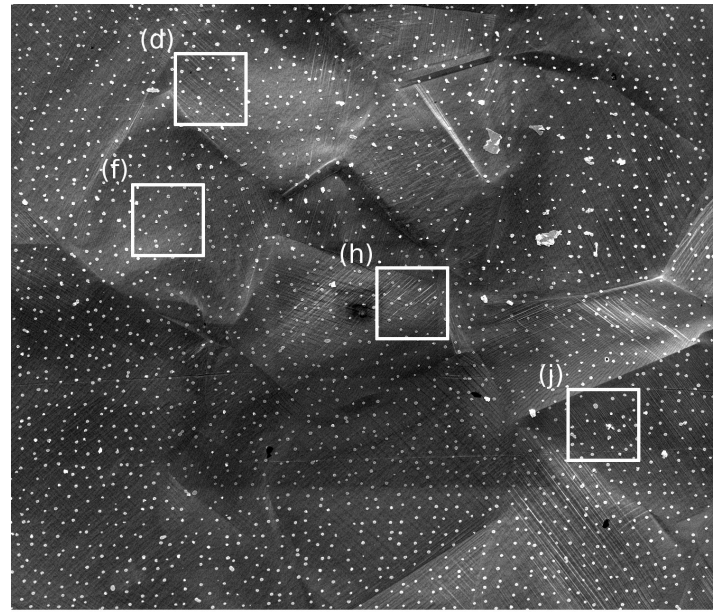

(b)

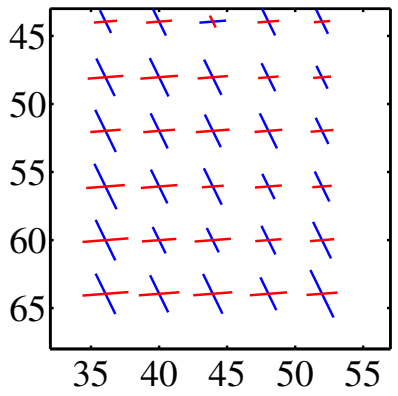

(e)

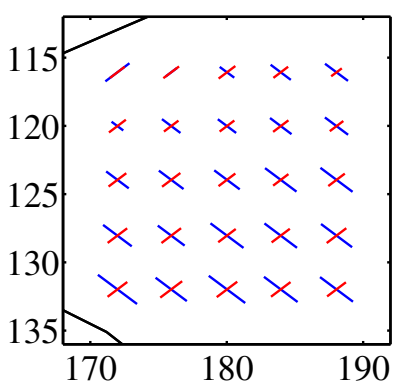

(i)

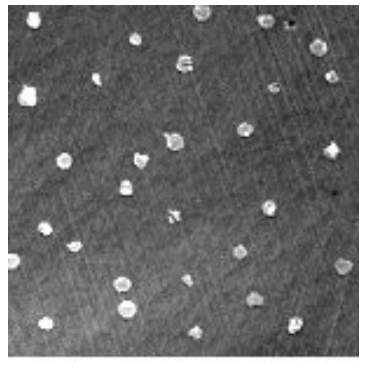

(f)

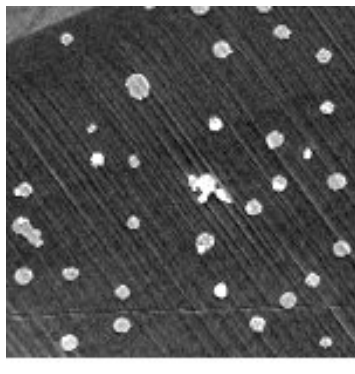

(j)

Figure 12: Intersection of the first (in red) and second (in blue) slip systems with the image plane $z=0$ drawn for a mean tensile strain of $4 \%$ (a). The length of the segments is proportional to the corresponding cumulative shear strain. The spatial axes are expressed in micrometers. The microstructure boundaries are shown as black lines on these fields. SE image of the region of interest acquired at the end of the tensile test and corrected with the measured displacement field (b). Four enlarged views of both figures are shown (c-j) 
for, a local evaluation of the stress is not sufficient to predict the local deformation. For instance, plastic strain is also triggered by strain accommodation conditions and slip transmission conditions at grain boundaries. In the context of simplified, fast and efficient calculation techniques, the results reported in this paper also suggest that mean-field homogenization models based on individual grains (e.g., self-consistent schemes) may overlook an important contribution to the macroscopic manifestation of plasticity and should be considered carefully. Most of the recent improvements in homogenization procedures have been made possible by the use of secondorder moment estimates either in composite materials [86] or in polycrystals [87], which are providing a more accurate effective response for complex loading paths without accounting for grain-to-grain interaction. Another way of improving the homogenization models is to consider intergranular kinematic incompatibilities [88] that may reveal mandatory to obtain satisfactory correlations with experiments at the grain scale.

The correlation between plastic strain and grain orientation is studied by assessing plastic glide along slip systems. It is shown that a few slip systems are simultaneously activated in each grain. A comparison between the experimental strain measurements and the results of a numerical simulation based on crystal plasticity with the same (surface) grain morphology and measured boundary conditions shows an excellent agreement for the major slip system and amplitude in each grain. However, some specific regions close to grain boundaries display sometimes significant differences and the slip traces are not well predicted in some grains or in the vicinity of some grain boundaries or triple junctions. This may either question the constitutive law used in the crystal plasticity modeling or result from the three-dimensional bulk morphology $[60,61,80]$ that remains inaccessible by resorting to SEM imaging without post-mortem (and tedious) 3DEBSD reconstructions based on serial sectioning [89]. An alternative method to obtain the 3D microstructure is diffraction contrast tomography [90] but it is currently limited by its resolution (minimum voxel dimension) by the maximum number of grains in the specimen and suffers in presence of lattice curvature among other issues. Although it is now possible to conduct studies with having access to the 3D microstructure, this is not yet possible for every material and several applications may consider only using a 2D information when possible and relevant. As previously observed [58] the correlations obtained with a macroscopic Schmid's factor are not 
satisfactory at the surface of general polycrystals but significant improvements are observed in this work when a more local approach is adopted.

Last, in this study the description of grain boundaries is simply defined as geometrical surfaces associated with a misorientation between grains and with no strain gradient dependence in the plasticity law accounting for GNDs as explored in several works [34, 37, 91]. A mechanical description in polycrystals using strain gradient plasticity together with specific constitutive laws for grain boundaries may be investigated in future studies.

\section{Acknowledgements}

The authors acknowledge the financial support of EDF within R\&D LOCO and PERFORM60 (www.perform60.net) projects. Michel Mahé, Nicolas Brynaert and the Electronic Microscopy Laboratory of Les Renardières are thanked for their help in preparing and conducting the SEM acquisitions. François Curtit, Ghiath Monnet, Nicolas Rupin and Charles Toulemonde are thanked for fruitful discussions. 


\section{AppendixA. Constitutive equations of the chosen crystal plasticity law and parameters}

The crystal plasticity model chosen in this study has been proposed by Méric et al. [77]. Its relationships are expressed for each of the 12 octahedral slip systems $s$ as

$$
\begin{gathered}
\dot{\gamma}_{s}=\dot{p}_{s} \frac{\tau_{s}-c \alpha_{s}}{\left|\tau_{s}-c \alpha_{s}\right|} \\
\dot{p}_{s}=\left\langle\frac{\left|\tau_{s}-c \alpha_{s}\right|-R_{s}\left(p_{s}\right)}{k}\right\rangle_{+}^{n} \\
R_{s}=R_{0}+q\left(\sum_{r=1}^{12} h_{s r}\left(1-e^{-b p_{r}}\right)\right) \\
\dot{\alpha}_{s}=\dot{\gamma}_{s}-d \alpha_{s} \dot{p}_{s}
\end{gathered}
$$

where $c, k, n, R_{0}, q, b, d$ are constitutive parameters, and $h_{s r}$ the coefficients of the interaction matrix between slip systems detailed in Table A.5. The brackets $\langle.\rangle_{+}$are the positive part of their argument. The values of the material-dependent parameters used in the simulations for the microstructure $A_{70}$ are given in Table A.6. 
Table A.5: Interaction matrix between slip systems. The nomenclature of slip systems is given in Table 4

\begin{tabular}{|l|l|l|l|l|l|l|l|l|l|l|l|l|}
\hline 1 & 1 & 2 & 3 & 4 & 5 & 6 & 7 & 8 & 9 & 10 & 11 & 12 \\
\hline 1 & $h_{1}$ & $h_{2}$ & $h_{2}$ & $h_{4}$ & $h_{5}$ & $h_{5}$ & $h_{5}$ & $h_{6}$ & $h_{3}$ & $h_{5}$ & $h_{3}$ & $h_{6}$ \\
\hline 2 \\
\hline 3
\end{tabular}

Table A.6: Values of the parameters of the crystal plasticity law [77] used in the study

\begin{tabular}{|c|c|c|c|c|c|c|c|c|c|c|c|c|}
\hline$n$ & $k$ & $R_{0}$ & $q$ & $b$ & $c$ & $d$ & $h_{1}$ & $h_{2}$ & $h_{3}$ & $h_{4}$ & $h_{5}$ & $h_{6}$ \\
\hline 11 & $12 \mathrm{MPa}^{-n} \mathrm{~s}^{-n}$ & $52 \mathrm{MPa}$ & $220 \mathrm{MPa}$ & 1.0 & $40 \mathrm{GPa}$ & $1.5 \mathrm{GPa}$ & 1.0 & 1.0 & 0.6 & 12.3 & 1.6 & 1.8 \\
\hline
\end{tabular}




\section{References}

[1] T. Jayakumar, M.D. Mathew, and K. Laha. High Temperature Materials for Nuclear Fast Fission and Fusion Reactors and Advanced Fossil Power Plants. Procedia Engineering, 55:259-270, 2013.

[2] M. Kamaya, and M. Itakura. Simulation for intergranular stress corrosion cracking based on a three-dimensional polycrystalline model. Engineering Fracture Mechanics, 76:386-401, 2009.

[3] M.D. McMurtrey, G.S. Was, L. Patrick, and D. Farkas. Relationship between localized strain and irradiation assisted stress corrosion cracking in an austenitic alloy. Materials Science and Engineering, 528:3730-3740, 2011.

[4] T. Couvant, L. Legras, T. Ghys, P. Gambier, N. Huin, and G. Ilevbare. Strain path effect on IGSCC initiation and oxidation of alloy 182 exposed to PWR primary water. in 15th International Conference on Environmental Degradation of Materials in Nuclear Power Systems-Water Reactors, Colorado Springs: TMS, 2011.

[5] O. Diard, S. Leclercq, G. Rousselier, and G. Cailletaud. Evaluation of finite element based analysis of 3D multicrystalline aggregates plasticity: Application to crystal plasticity model identification and the study of stress and strain fields near grain boundaries. International Journal of Plasticity, 21:691-722, 2005.

[6] A. Musienko, and G. Cailletaud. Simulation of inter- and transgranular crack propagation in polycrystalline aggregates due to stress corrosion cracking. Acta Materialia, 57:3840 3855, 2009.

[7] T.R. Bieler, P. Eisenlohr, F. Roters, D. Kumar, D.E. Mason, M.A. Crimp, and D. Raabe. The role of heterogeneous deformation on damage nucleation at grain boundaries in single phase metals. International Journal of Plasticity, 25:1655-1683, 2009.

[8] I. Simonovski, and L. Cizelj. Cohesive element approach to grain level modelling of intergranular cracking. Engineering Fracture Mechanics, 110:364-377, 2013. 
[9] R.A. Lebensohn, R. Brenner, O. Castelnau, and A.D. Rollett. Orientation image-based micromechanical modelling of subgrain texture evolution in polycrystalline copper. Acta Materialia 56:3914-3926, 2008.

[10] R.A. Lebensohn, A.K. Kanjarla, and P. Eisenlohr. An elasto-viscoplastic formulation based on fast Fourier transforms for the prediction of micromechanical fields in polycrystalline materials. International Journal of Plasticity 32-33:59-69, 2012.

[11] A.K. Kanjarla, R.A. Lebensohn, L. Balogh, and C.N. Tomé. Study of internal lattice strain distributions in stainless steel using a full-field elasto-viscoplastic formulation based on fast Fourier transforms. Acta Materialia, 60:3094-3106, 2012.

[12] R. Brenner, and P. Suquet. Overall response of viscoelastic composites and polycrystals: exact asymptotic relations and approximate estimates. International Journal of Solids and Structures, 50:1824-1838, 2013.

[13] P. Eisenlohr, M. Diehl, R.A. Lebensohn, and F. Roters. A spectral method solution to crystal elasto-viscoplasticity at finite strains. International Journal of Plasticity 46:37-53, 2013.

[14] F. Roters, P. Eisenlohr, L. Hantcherli, D.D. Tjahjanto, T.R. Bieler, and D. Raabe. Overview of constitutive laws, kinematics, homogenization and multiscale methods in crystal plasticity finite-element modeling: Theory, experiments, applications. Acta Materialia, 58:1152$1211,2010$.

[15] L. Priester. "Dislocation-interface" interaction - stress accommodation processes at interfaces. Materials Science and Engineering: A, 309-310:430-439, 2001.

[16] J. Jiang, T.B. Britton, and A.J. Wilkinson. Measurement of geometrically necessary dislocation density with high resolution electron backscatter diffraction: Effects of detector binning and step size. Ultramicroscopy, 125:1-9, 2013.

[17] T. Ohashi, R.I. Barabash, J.W.L. Pang, G.E. Ice, and O.M. Barabash. X-ray microdiffraction and strain gradient crystal plasticity studies of geometrically necessary dislocations near a Ni bicrystal grain boundary. International Journal of Plasticity 25:920-941, 2009. 
[18] C. Perrin, S. Berbenni, H. Vehoff, and M. Berveiller. Role of discrete intragranular slip on lattice rotations in polycrystalline Ni: Experimental and micromechanical studies. Acta Materialia, 58:4639-4649, 2010.

[19] N.A. Fleck, and J.W. Hutchinson. A phenomenological theory for strain gradient effects in plasticity. Journal of the Mechanics and Physics of Solids, 41:1825-1857, 1993.

[20] H. Gao, Y. Huang, W.D. Nix, and J.W. Hutchinson. Mechanism-based strain gradient plasticity - I. Theory. Journal of the Mechanics and Physics of Solids, 47:1239-1263, 1999.

[21] Z. Xue, Y. Huang, and M. Li. Particle size effect in metallic materials: a study by the theory of mechanism-based strain gradient plasticity. Acta Materialia, 50:149-160, 2002.

[22] M.E. Gurtin. A gradient theory of single-crystal viscoplasticity that accounts for geometrically necessary dislocations. Journal of the Mechanics and Physics of Solids, 50:5-32, 2002.

[23] S. Forest, F. Barbe, and G. Cailletaud. Cosserat modelling of size effects in the mechanical behaviour of polycrystals and multi-phase materials. International Journal of Solids and Structures, 37:7105-7126, 2000.

[24] P. Neff. A finite-strain elastic-plastic Cosserat theory for polycrystals with grain rotations. International Journal of Engineering Science, 44:574-594, 2006.

[25] B. Klusemann, and T. Yalçinkaya. Plastic deformation induced microstructure evolution through gradient enhanced crystal plasticity based on a non-convex Helmholtz energy. International Journal of Plasticity 48:168-188, 2013.

[26] J.R. Mayeur, and D.L. McDowell. A comparison of Gurtin type and micropolar theories of generalized single crystal plasticity. International Journal of Plasticity 57:29-51, 2014.

[27] M.F. Ashby. The deformation of plastically non-homogeneous alloys. Philosophical Magazine, 21:399-424, 1970. 
[28] A. Acharya, and A.J. Beaudoin. Grain-size effect in viscoplastic polycrystals at moderate strains. Journal of the Mechanics and Physics of Solids, 48:2213-2230, 2000.

[29] A.J. Beaudoin, A. Acharya, S.R. Chen, D.A. Korzekwa, and M.G. Stout. Consideration of grain-size effect and kinetics in the plastic deformation of metal polycrystals. Acta Materialia, 48:3409-3423, 2000.

[30] M.G.D. Geers, W.A.M. Brekelmans, and P.J.M. Janssen. Size effects in miniaturized polycrystalline $\{\mathrm{FCC}\}$ samples: Strengthening versus weakening. International Journal of Solids and Structures, 43:7304-7321, 2006.

[31] A.G. Evans, and J.W. Hutchinson. A critical assessment of theories of strain gradient plasticity. Acta Materialia, 57:1675-1688, 2009.

[32] C. Keller, E. Hug, A.M. Habraken, and L. Duchene. Finite element analysis of the free surface effects on the mechanical behavior of thin nickel polycrystals. International Journal of Plasticity, 29:155-172, 2012.

[33] B.W. Baker, T.R. McNelley and, L.N. Brewer. Grain size and particle dispersion effects on the tensile behavior of friction stir welded \{MA956\} oxide dispersion strengthened steel from low to elevated temperatures. Materials Science and Engineering: A, 589:217-227, 2014.

[34] N.M. Cordero, S. Forest, E.P. Busso, S. Berbenni, and M. Cherkaoui. Grain size effects on plastic strain and dislocation density tensor fields in metal polycrystals. Computational Materials Science, 52:7-13, 2012.

[35] K.S. Cheong, E.P. Busso, and A. Arsenlis. A study of microstructural length scale effects on the behaviour of $\{\mathrm{FCC}\}$ polycrystals using strain gradient concepts. International Journal of Plasticity, 21:1797-1814, 2005.

[36] V. Taupin, S. Berbenni, C. Fressengeas, and O. Bouaziz. On particle size effects: An internal length mean field appraoch using field dislocation mechanics. Acta Materialia, 58:5532-5544, 2010. 
[37] V. Taupin, S. Berbenni, and C. Fressengeas. Size effects on the hardening of channel-type microstructures: A field dislocation mechanics-based approach. Acta Materialia, 60:664$673,2012$.

[38] H.-J. Chang, A. Gaubert, M. Fivel, S. Berbenni, O. Bouaziz, and M. Cherkaoui. Analysis of particle induced dislocation structures using three-dimensional dislocation dynamics and strain gradient plasticity. Computational Materials Science, 52:33-39, 2012.

[39] E. Héripré, M. Dexet, J. Crépin, L. Gélébart, A. Roos, M. Bornert, and D. Caldemaison. Coupling between experimental measurements and polycrystal finite element calculations for micromechanical study of metallic materials. International Journal of Plasticity, 23:1512-1539, 2007.

[40] A. Ma, F. Roters, and D. Raabe. On the consideration of interactions between dislocations and grain boundaries in crystal plasticity finite element modeling - Theory, experiments, and simulations. Acta Materialia, 54:2181-2194, 2006.

[41] A. Ma, F. Roters, and D. Raabe. Studying the effect of grain boundaries in dislocation density based crystal-plasticity finite element simulations. International Journal of Solids and Structures, 43:7287-7303, 2006.

[42] E. Soppa, P. Doumalin, P. Binkele, T. Wiesendanger, M. Bornert, and S. Schmauder. Experimental and numerical characterisation of in-plane deformation in two-phase materials. Computational Materials Science, 21:261-275, 2001.

[43] M.A. Sutton, N. Li, D.C. Joy, A.P. Reynolds, and X. Li. Scanning electron microscopy for quantitative small and large deformation measurements part i: Sem imaging at magnifications from 200 to 10.000. Experimental Mechanics, 47:775-787, 2007.

[44] M.A. Sutton, N. Li, D. Garcia, N. Cornille, J.-J. Orteu, S.R. McNeill, H.W. Schreier, X. Li, and A.P. Reynolds. Scanning electron microscopy for quantitative small and large deformation measurements part ii: Experimental validation for magnifications from 200 to 10.000 . Experimental Mechanics, 47:789-804, 2007. 
[45] M.A. Tschopp, B.B. Bartha, W.J. Porter, P.T. Murray, and S.B. Fairchild. Microstructuredependent local strain behavior in polycrystals through in situ scanning electron microscope tensile experiments. Metallurgical Transactions A, 40:2363-2368, 2009.

[46] A.D. Kammers, and S. Daly. Digital Image Correlation under Scanning Electron Microscopy: Methodology and Validation. Experimental Mechanics, 53:1746-1761, 2013.

[47] W.Z. Abuzaid, M.D. Sangid, J.D. Carroll, H. Sehitoglu, and J. Lambros. Slip transfer and plastic strain accumulation across grain boundaries in Hastelloy X. Journal of the Mechanics and Physics of Solids, 60:1201-1220, 2012.

[48] J.L.W. Carter, M.W. Kuper, M.D. Uchic, and M.J. Mills. Characterization of localized deformation near grain boundaries of superalloy René-104 at elevated temperature. Materials Science and Engineering: A, 605:127-136, 2014.

[49] H. Jin, W.-Y. Lu, and J. Korellis. Micro-scale deformation measurement using the digital image correlation technique and scanning electron microscope imaging. Journal of Strain Analysis for Engineering Design, 43:719-728, 2008.

[50] H. Jin, W.-Y. Lu, S. Haldar, and H.A. Bruck. Microscale characterization of granular deformation near a crack tip. Journal of Materials Science, 46:6596-6602, 2011.

[51] S.R. McNeill, W.H. Peters, and M.A. Sutton. Estimation of stress intensity factor by digital image correlation. Engineering Fracture Mechanics, 28:101-112, 1987.

[52] J.D. Carroll, W. Abuzaid, J. Lambros, and H. Sehitoglu. High resolution digital image correlation measurements of strain accumulation in fatigue crack growth. International Journal of Fatigue, 57:140-150, 2013.

[53] C.C. Tasan, M. Diehl, D. Yan, C. Zambaldi, P. Shanthraj, F. Roters, and D. Raabe. Integrated experimental-simulation analysis of stress and strain partitioning in multiphase alloys. Acta Materialia, 81:386-400, 2014. 
[54] G. Martin, C.W. Sinclair, and R.A. Lebensohn. Microscale plastic strain heterogeneity in slip dominated deformation of magnesium alloy containing rare earth. Materials Science and Engineering: A, 603:37-51, 2014.

[55] C.C. Tasan, J.P.M., Hoefnagels, M. Diehl, D. Yan, F. Roters, and D. Raabe. Strain localization and damage in dual phase steels investigated by coupled in-situ deformation experiments and crystal plasticity simulations. International Journal of Plasticity, 63:198-210, 2014.

[56] H. Lim, J.D. Carroll, C.C. Battaile, T.E. Buchheit, B.L. Boyce, and C.R. Weinberger. Grainscale experimental validation of crystal plasticity finite element simulations of tantalum oligocrystals. International Journal of Plasticity, 60:1-18, 2014.

[57] H. Lim, J.D. Carroll, C.C. Battaile, B.L. Boyce, and C.R. Weinberger. Quantitative comparison between experimental measurements and CP-FEM predictions of plastic deformation in a tantalum oligocrystal. International Journal of Plasticity, 92:98-108, 2015.

[58] J.D. Carroll, B.G. Clark, T.E. Buchheit, B.L. Boyce, and C.R. Weinberger. An experimental statistical analysis of stress projection factors in BCC tantalum. Materials Science and Engineering: A, 581:108-118, 2013.

[59] H. Li, D.E. Mason, T.R. Bieler, C.J. Boehlert, and M.A.Crimp. Methodology for estimating the critical resolved shear stress ratios of $\alpha$-phase Ti using EBSD-based trace analysis. Acta Materialia, 61:7555-7567, 2013.

[60] A. Zeghadi, F. N'Guyen, S. Forest, A.-F. Gourgues, and O. Bouaziz. Ensemble averaging stress-strain fields in polycrystalline aggregates with a constrained surface microstructure Part 1: Anisotropic elastic behaviour. Philosophical Magazine, 87:1401-1424, 2007.

[61] A. Zeghadi, S. Forest, A.-F. Gourgues, and O. Bouaziz. Ensemble averaging stress-strain fields in polycrystalline aggregates with a constrained surface microstructure - Part 2: crystal plasticity. Philosophical Magazine, 87:1425-1446, 2007. 
[62] M. Montagnat, O. Castelnau, P.D. Bons, S.H. Faria, O. Gagliardini, F. Gillet-Chaulet, F. Grennerat, A. Griera, R.A Lebensohn, H. Moulinec, J. Roessiger, and P. Suquet. Multiscale modeling of ice deformation behavior. Journal of Structural Geology, 61:78-108, 2014.

[63] J. Schwartz, O. Fandeur, and C. Rey. Fatigue crack initiation modeling of 316LN steel based on non local plasticity theory. Procedia Engineering, 2:1353-1362, 2010.

[64] G.V. Prasad Reddy, C. Robertson, C. Déprés, and M. Fivel. Effect of grain disorientation on stage I fatigue crack retardation in FCC poly-crystals: a three dimensional dislocation dynamics investigation. Acta Materialia, 61:5300-5310, 2013.

[65] A. Guery, F. Latourte, F. Hild, and S. Roux. Characterization of SEM speckle pattern marking and imaging distortion by digital image correlation. Measurement Science and Technology, 25:12pp, 2014.

[66] H. Leclerc, J.N. Périé, S. Roux, and F. Hild. Integrated digital image correlation for the identification of mechanical properties. MIRAGE 2009, LNCS, 5496:161-171, 2009.

[67] Z. Tomičević, F. Hild, and S. Roux. Mechanics-aided digital image correlation. Journal of Strain Anal. Eng. Des., 48:330-343, 2013.

[68] G. Besnard, F. Hild, and S. Roux. "Finite-Element" displacement fields analysis from digital images: Application to Portevin-Le Châtelier bands. Experimental Mechanics, 46:789_ 803, 2006.

[69] J. Réthoré, F. Hild, and S. Roux. Shear-band capturing using a multiscale extended digital image correlation technique. Computer Methods in Applied Mechanics and Engineering, 196:5016-5030, 2007.

[70] J. Réthoré, G. Besnard, G. Vivier, F. Hild, and S. Roux. Solutions of ill-posed problems. Philosophical Magazine, 88:3339-3355, 2008. 
[71] A.N. Tikhonov, and V.Y. Arsenin. Solutions of ill-posed problems. J. Wiley, New York (USA), 1977.

[72] S. Roux, F. Hild, and H. Leclerc. Mechanical Assistance to DIC. Procedia IUTAM, 4:159$168,2012$.

[73] K. Kunze, S.I. Wright, B.L. Adams, and D.J. Dingley. Advances in automatic EBSP single orientation measurements. Textures and Microstructures, 20:41-54, 1993.

[74] G. Nolze. Image distortions in SEM and their influences on EBSD measurements. Ultramicroscopy, 107:172-183, 2007.

[75] N.J. Petch. The cleavage strength of polycrystals. Journal of the Iron and Steel Institute, 174:25-28, 1953.

[76] http://www.code-aster.org/.

[77] L. Méric, P. Poubanne, and G. Cailletaud. Single crystal modeling for structural calculations: Part 1 - Model Presentation. Journal of Engineering Materials and Technology, 113:162-170, 1991.

[78] M. Berveiller, and A. Zaoui. An extension of the self-consistent scheme to plastically flowing polycrystal. Journal of the Mechanics and Physics of Solids, 26:325-344, 1979.

[79] Y. Guilhem, S. Basseville, F. Curtit, J.-M. Stéphan, and G. Cailletaud. Investigation of the effect of grain clusters on fatigue crack initiation in polycrystals. International Journal of Fatigue, 32:1748-1763, 2010.

[80] C. Zhang, H. Li, P. Eisenlohr, W. Liu, C.J. Boehlert, M.A. Crimp, and T.R. Bieler. Effect of realistic 3D microstructure in crystal plasticity finite element analysis of polycrystalline Ti-5Al-2.5Sn. International Journal of Plasticity, 69:21-35, 2015.

[81] P. Villechaise, L. Sabatier, and J.C. Girard. On slip band features and crack initiation in fatigued 316L austenitic stainless steel: Part 1: Analysis by electron back-scattered diffrac- 
tion and atomic force microscopy. Materials Science and Engineering: A, 323:377-385, 2002.

[82] J. Miao, T.M. Pollock, and J.W. Jones. Crystallographic fatigue crack initiation in nickelbased superalloy René 88DT at elevated temperature. Acta Materialia, 57:5964-5974, 2009.

[83] B. Klusemann, B. Svendsen, and H. Vehoff. Modeling and simulation of deformation behavior, orientation gradient development and heterogeneous hardening in thin sheets with coarse texture. International Journal of Plasticity, 50:109-126, 2013.

[84] F. Latourte, N. Rupin, M. He, A. Parrot, and S. Leclercq. Full field measurements used for assessing industrial issues - two examples. Procedia IUTAM, 4:92-105, 2012.

[85] Y. Yang, L. Wang, T.R. Bieler, P. Eisenlohr, and M.A. Crimp. Quantitative AFM characterization and crystal plasticity finite element modeling of heterogeneous deformation in commercial purity titanium. Metallurgical and Materials Transactions A, 42:636-644, 2011.

[86] N. Lahellec, and P. Suquet. Effective response and field statistics in elasto-plastic and elasto-viscoplastic composites under radial and non-radial loadings. International Journal of Plasticity, 42:1-30, 2013.

[87] Y. Liu, and P.P. Castañeda. Second-order theory for the effective behavior and field fluctuations in viscoplastic polycrystals. Journal of the Mechanics and Physics of Solids, 52:467495, 2004.

[88] D.D. Tjahjanto, P. Eisenlohr, and F. Roters. A novel grain cluster-based homogenization scheme. Modelling and Simulation in Materials Science and Engineering, 52:21pp, 2010.

[89] J. Schwartz, O. Fandeur, and C. Rey. Numerical approach of cyclic behaviour of 316LN stainless steel based on a polycrystal modelling including strain gradients. International Journal of Fatigue, 55:202-212, 2013. 
[90] A. King, G. Johnson, D. Engelberg, W. Ludwig, and J. Marrow. Observations of intergranular stress corrosion cracking in a grain-mapped polycrystal. Science, 321:382-385, 2008.

[91] T. Mayama, T. Ohashi, and R. Kondou. Geometrically necessary dislocation structure organization in FCC bicrystal subjected to cyclic plasticity. International Journal of Plasticity, 25:2122-2140, 2009. 